\title{
On the Existence of Radiation Gauges in Petrov type II spacetimes
}

\author{
Larry R. Price†, Karthik Shankar $\ddagger$, Bernard F. Whiting 9 \\ Department of Physics, University of Florida, PO Box 118440, Gainesville, FL 32611, \\ USA \\ E-mail: †price@phys.ufl.edu, $\nmid$ karthik@phys .ufl .edu, $\llbracket$ bernard@phys . ufl .edu
}

\begin{abstract}
The radiation gauges used by Chrzanowski (his IRG/ORG) for metric reconstruction in the Kerr spacetime seem to be over-specified. Their specification consists of five conditions: four, which we treat here as valid gauge conditions, plus an additional condition on the trace of the metric perturbation. In this work, we utilize a newly developed form of the perturbed Einstein equations to establish a condition on a particular tetrad component of the stress-energy tensor - under which the full IRG/ORG can be imposed. Using gauge freedom, we are able to impose the full IRG for Petrov type II and type D backgrounds, using a different tetrad for each case. As a specific example, we work through the process of imposing the IRG in a Schwarzschild background, using a more traditional approach. Implications for metric reconstruction using the Teukolsky curvature perturbations in type D spacetimes are briefly discussed.
\end{abstract}




\section{Introduction}

The Regge-Wheeler[20] (RW) approach to perturbations of the Schwarzschild spacetime is usually understood to lead, by direct integration, to perturbations for all parts of the metric in terms of gauge invariant quantities[13]. In fact, it has been evident for a long time[19] that the RW variable actually represents part of the perturbation of the Weyl curvature (namely, $\operatorname{Im}\left(\Psi_{2}\right)[15]$; see [9] for a clear demonstration of this and [28] for further discussion). These contrasting perspectives are reconciled by the exceptional fact that the geometrical symmetries of the Schwarzschild spacetime permit an analysis virtually transparent to both angular and time derivatives.

For the perturbations of the Kerr spacetime, the situation is completely different. Instead of the RW equation, we have the Teukolsky equation for the gauge (and tetrad) invariant parts $\left(\Psi_{0}\right.$ and $\Psi_{4}$ ) of the perturbed Weyl curvature. To date, metric reconstruction[16, 12] can then be performed by a (Hertz) potential method championed by Chrzanowski[1], but only fully in vacuum, and even so, only in a special class of over-specified gauges referred to as "radiation gauges". We expect that Hertz potential methods are set to play an increasingly key rôle as we pursue deeper studies of perturbations of Petrov type D spacetimes. However, neither Chrzanowski's analysis, nor the more general analyses of Cohen and Kegeles[2, 10] and Stewart[23] for perturbations of Petrov type II spacetimes, spells out the precise circumstances in which radiation gauges are able to exist. $\dagger$ The purpose of this paper is to address and dispel this concern, by specifying exactly when a radiation gauge may be imposed.

The Petrov classification refers to the properties of the (always null) eigenvectors of the Weyl tensor, referred to as principle null directions (PNDs). In a type II spacetime, one of these PNDs is repeated. It may be either the ingoing null vector $l^{a}$ or the outgoing null vector $n^{a}$. In a type D spacetime, two of the PNDs are repeated, namely, both $l^{a}$ and $n^{a}$. Radiation gauges have been defined[1] with respect to either one of these PNDs. In a suitable spacetime, with metric $g_{a b}$ and for metric perturbation $h_{a b}, \ddagger$ the gauge conditions specified for a radiation gauge are either:

i) $l^{a} h_{a b}=0$ and $g^{a b} h_{a b}=0$, referred to as "Ingoing" (IRG), or

ii) $n^{a} h_{a b}=0$ and $g^{a b} h_{a b}=0$, referred to as "Outgoing" (ORG).

In each case, since these represent five distinct conditions, it is clear that radiation gauges cannot be defined in general, but it turns out they can be prescribed in special circumstances, which we have investigated here.

In general terms, in type D background spacetimes, two radiation gauges are indeed possible. In type II background spacetimes, depending on which principle null direction is repeated, only one or the other of these gauges would be possible. In all cases, we find that radiation gauges can normally actually exist only for a perturbed stress tensor, $\mathcal{T}_{a b}$, satisfying $\mathcal{T}_{a b} l^{a} l^{b} \equiv \mathcal{T}_{l l}=0$ in the IRG case or $\mathcal{T}_{a b} n^{a} n^{b} \equiv \mathcal{T}_{n n}=0$ in the ORG

$\dagger$ However, the constructive procedure of Stewart does go a long way in this direction.

$\ddagger$ Throughout, we have consistently used the perturbed metric to be $g_{a b}-h_{a b}$. 
case. This is because, in the appropriate circumstances, the $l l$ - (or $n n-$ ) component of the perturbed Einstein equation depends only on the trace of the metric perturbation, which cannot be zero if the source for that equation is non-zero. Remarkably, in all type II background spacetimes (which include type D as a special case), a non-zero solution to the trace equation without sources can be fully gauged away by the use of residual gauge freedom.

The layout of the paper is as follows. We first introduce a new form of the perturbed Einstein equations in the Newman-Penrose formalism. Then, we describe the radiation gauges in more detail, followed by explanations of how they are set up in type II and type D background spacetimes, respectively. This requires us to examine the perturbed Einstein tensor to understand fully the implications of attempting to impose a radiation gauge. We illustrate the residual gauge freedom and the condition for it to remove the trace of the perturbed metric. Next, we demonstrate the implication of our analysis in the Schwarzschild spacetime. Finally we contrast the construction of Hertz potentials and the construction of radiation gauges.

\section{A new form of the perturbed Einstein equations}

We choose to work with a formulation of the perturbed Einstein equations that makes explicit use of the modified Newman-Penrose[14] (NP) formalism of Geroch, Held and Penrose[4] (GHP). For a detailed explanation of the GHP formalism see also [17]. The starting point is to take a complex null tetrad $\left\{l^{a}, n^{a}, m^{a}, \bar{m}^{a}\right\}$, where an overbar denotes the complex conjugate, normalized so that $\S$

$$
l^{a} n_{a}=-m^{a} \bar{m}_{a}=1 .
$$

Then, the spacetime metric has the following expression:

$$
g_{a b}=2 l_{(a} n_{b)}-2 m_{(a} \bar{m}_{b)},
$$

in which round brackets, (), around indices denote symmetrization. Note that the metric is invariant under the transformation

$$
\begin{array}{ll}
l^{a} \rightarrow \zeta \bar{\zeta} l^{a}, & n^{a} \rightarrow \zeta^{-1} \bar{\zeta}^{-1} n^{a}, \\
m^{a} \rightarrow \zeta \bar{\zeta}^{-1} m^{a}, & \bar{m}^{a} \rightarrow \zeta^{-1} \bar{\zeta}^{a},
\end{array}
$$

for some complex number $\zeta$. A key feature of the GHP formalism is that all objects of interest transform homogeneously under (3). A quantity, $\chi$, is said to be of type $\{p, q\}$ if under (3) it transforms as $\chi \rightarrow \zeta^{p} \bar{\zeta}^{q} \chi$. Alternatively, it useful to speak of the spin-weight $s=(p-q) / 2$ and boost-weight $b=(p+q) / 2$ of $\chi$. A table listing the types of the fundamental GHP quantities can be found in [4].

$\S$ The conventions displayed in (1) and (2) with signature [+,-,-,-] are characteristic of the NP formalism. 
We can express the metric perturbation, $h_{a b}$, in terms of the tetrad vectors according to

$$
\begin{aligned}
h_{a b}= & h_{n n} l_{a} l_{b}-2 h_{n \bar{m}} l_{(a} m_{b)}-2 h_{n m} l_{(a} \bar{m}_{b)}+2 h_{l n} l_{(a} n_{b)} \\
& +h_{l l} n_{a} n_{b}-2 h_{l \bar{m}} n_{(a} m_{b)}-2 h_{l m} n_{(a} \bar{m}_{b)} \\
& +h_{m m} \bar{m}_{a} \bar{m}_{b}+2 h_{m \bar{m}} m_{(a} \bar{m}_{b)}+h_{\bar{m} \bar{m}} m_{a} m_{b},
\end{aligned}
$$

where $h_{l l}=h_{a b} l^{a} l^{b}, h_{l m}=h_{a b} l^{a} m^{b}$ and so on, are the tetrad components of the metric perturbation. Note that the GHP type of each component of the metric perturbation is inherited from the tetrad vectors, e.g. $h_{l l}$ has type $\{2,2\}, h_{n \bar{m}}$ has type $\{-2,0\}$, etc. The perturbed Einstein tensor, $\mathcal{E}_{a b}$, is then computed via

$\mathcal{E}_{a b}=-\frac{1}{2} \Theta^{c} \Theta_{c} h_{a b}-\frac{1}{2} \Theta_{a} \Theta_{b} h_{c}^{c}+\Theta^{c} \Theta_{(a} h_{b) c}+\frac{1}{2} g_{a b}\left(\Theta^{c} \Theta_{c} h_{d}^{d}-\Theta^{c} \Theta^{d} h_{c d}\right)$,

where

$$
\Theta_{a}=l_{a} \mathrm{P}^{\prime}+n_{a} \mathrm{P}-m_{a} \partial^{\prime}-\bar{m}_{a} \partial,
$$

is just the covariant derivative expressed in GHP language. We can use the expression in (6) to define the GHP derivatives 'thorn' ( $\left.\mathrm{P}=l^{a} \Theta_{a}\right)$, 'edth' $\left(ð=m^{a} \Theta_{a}\right)$ and their 'primes' ( $\mathrm{P}^{\prime}=n^{a} \Theta_{a}$ and $\left.\partial^{\prime}=\bar{m}^{a} \Theta_{a}\right)$. These derivative operators also inherit their type from the corresponding tetrad vectors:

$$
\begin{array}{ll}
\text { Р : }\{1,1\}, & \mathrm{P}^{\prime}:\{-1,-1\}, \\
\text { ð : }\{1,-1\}, & \partial^{\prime}:\{-1,1\} .
\end{array}
$$

It is sometimes useful to think of $\mathrm{P}\left(\mathrm{P}^{\prime}\right)$ and $\partial\left(\chi^{\prime}\right)$ as boost and spin raising (lowering) operators, respectively. With these conventions, the perturbed Einstein equations are given in general as

$$
\mathcal{E}_{a b}=8 \pi \mathcal{T}_{a b},
$$

where $\mathcal{T}_{a b}$ is the stress-energy tensor source for the perturbation.

The form we use for the perturbed Einstein equations offers several advantages. First, it allows us to deal with perturbations of an entire class of spacetimes at once, say of Petrov type II or type D. Furthermore, this level of generality comes at no expense in terms of the complexity of the equations. See, for example, Appendix A which lists the components of the perturbed Einstein tensor for an arbitrary type II background. Additionally, the requirement that every term in an expression have the same spin- and boost- weight provides both a useful check on the equations and new insight into the structure of the perturbed Einstein equations. All of this comes with the added benefit of having a simple and straightforward way of dealing with gauge freedom, which has proved to be crucial for describing metric perturbations. We find this form of the equations provides a powerful new tool for exploring metric perturbations.

\section{The Radiation Gauges}

The ingoing radiation gauge (IRG) is a crucial ingredient for the reconstruction of metric perturbations of Petrov type D spacetimes from curvature perturbations. They 
first appear, unexplained, in the work of Cohen and Kegeles [2] (for perturbations of Petrov type II spacetimes) and Chrzanowski [1] (who considered perturbations of Petrov type D spacetimes), but the work that comes closest to our contribution in describing their origin is that of Stewart [23], again for the more general case of type II spacetimes.

In type II background spacetimes, the IRG is defined by the conditions

$$
\begin{aligned}
& l^{a} h_{a b}=0, \\
& g^{a b} h_{a b}=0,
\end{aligned}
$$

where $l^{a}$ is aligned with the repeated PND of the background Weyl tensor. If $n^{a}$ rather than $l^{a}$ is a repeated PND, we instead define the outgoing radiation gauge (ORG) by

$$
\begin{aligned}
& n^{a} h_{a b}=0, \\
& g^{a b} h_{a b}=0 .
\end{aligned}
$$

In type II background spacetimes, only one or the other of these options exists (IRG or ORG), whereas in Petrov type D background spacetimes, there is the possibility of defining both gauges. For the remainder of this work we focus on the IRG. Results for the ORG can be obtained by making the replacement $l^{a} \leftrightarrow n^{a}$.

Equations $(9 a-b)$ translate into algebraic conditions on the components of the metric perturbation. We will refer to the four conditions in $(9 a)$ as the $l \cdot h$ gauge conditions. $\|$ In terms of the tetrad components of the metric perturbation, these gauge conditions read:

$$
h_{l l}=0, \quad h_{l n}=0, \quad h_{l m}=0, \quad h_{l \bar{m}}=0 .
$$

The condition in $(9 b)$ will be referred to as the trace condition and can be expressed in terms of the components of the metric perturbation as $h_{l n}-h_{m \bar{m}}=0$, which, when (11) is imposed, simply reads

$$
h_{m \bar{m}}=0 .
$$

Because the IRG constitutes a total of five conditions on the metric perturbation, instead of the four one might expect for a gauge condition, it is necessary to ensure that the extra condition does not interfere with any physical degree of freedom in the problem, such as one coming from a source. The importance of this consideration can be seen immediately from (A.1) of Appendix A, in which every term would be removed by (11) and (12), rendering (A.1) inoperable whenever it has a non-zero source. In the next section we will look to the perturbed Einstein equations to determine the circumstances under which we can safely impose all five of the conditions that constitute the IRG.

It is useful to note the similarity between the full IRG, $(9 a-b)$, and the more commonly known transverse traceless (TT) gauge defined by

$$
\nabla^{a} h_{a b}=0, \quad g^{a b} h_{a b}=0,
$$

I| Recently, when applied specifically to the Schwarzschild spacetime, these conditions were given a geometrical interpretation, and referred to as light-cone gauge conditions[18], though they are not the conditions originally introduced for gravitation with that name[21]. It may well be that this description is suitable more generally, although presumably without the specific geometrical interpretation of [18]. 
which, at a first glance, also appears to be over-specified. In fact, the TT gauge exists for any vacuum perturbation of an arbitrary, globally hyperbolic, vacuum solution[26], because imposing the differential part of the gauge does not exhaust all of the available gauge freedom. Interestingly enough, Stewart's analysis in terms of Hertz potentials[23] begins by considering a metric perturbation in the TT gauge. However, in order to construct the curved space analogue of a Hertz potential, he is compelled to perform a transformation that destroys (13) and instead yields a metric perturbation in the IRG. $\uparrow$ Furthermore it appears that the restriction to type II spacetimes is essential for Stewart's analysis. From these observations, we expect radiation gauges to exist under conditions less general than those required for the existence of the TT gauge. At the same time, we should not be surprised that the IRG inherits the feature of residual gauge freedom.

Consider a gauge transformation on the metric perturbation generated by a gauge vector, $\xi_{a}$. To create a transformed metric in the $l \cdot h$ gauge, the gauge conditions (11) require

$$
l^{a}\left(h_{a b}-\xi_{(a ; b)}\right)=0,
$$

where the semicolon denotes the covariant derivative. In terms of components this reads

$$
\begin{array}{ll}
2 \mathrm{P} \xi_{l} & =h_{l l}, \\
\mathrm{P}^{\prime} \xi_{l}+\mathrm{P} \xi_{n}+\left(\tau+\bar{\tau}^{\prime}\right) \xi_{\bar{m}}+\left(\bar{\tau}+\tau^{\prime}\right) \xi_{m} & =h_{l n}, \\
(\mathrm{P}+\bar{\rho}) \xi_{m}+\left(ð+\bar{\tau}^{\prime}\right) \xi_{l} & =h_{l m}, \\
(\mathrm{P}+\rho) \xi_{\bar{m}}+\left(\partial^{\prime}+\tau^{\prime}\right) \xi_{l} & =h_{l \bar{m}} .
\end{array}
$$

Similarly, for the trace condition (12) to be satisfied by the gauge transformed metric, we require

$$
\chi^{\prime} \xi_{m}+ð \xi_{\bar{m}}+\left(\rho^{\prime}+\bar{\rho}^{\prime}\right) \xi_{l}+(\rho+\bar{\rho}) \xi_{n}=h_{m \bar{m}} .
$$

Any extra gauge transformation that satisfies $l^{a} \xi_{(a ; b)}=0$, that is, solves the homogeneous form of (15), preserves the four $l \cdot h$ gauge conditions (11). This is what is meant by residual gauge freedom. We will explicitly use this residual gauge freedom to impose the $l \cdot h$ and trace conditions simultaneously, thus establishing the IRG. We will find that some gauge freedom still remains, as explained in section 4.1.

Now, we turn our attention to the general case of type II background spacetimes.

\section{Imposing the IRG in type II}

In order to show that residual gauge freedom can be used to impose the IRG, we need to solve for the residual gauge freedom as well as examine any perturbed Einstein equation that might impede the imposition of the trace condition of the IRG. For this, we turn to a coordinate-free integration method develop by Held. Rather than give a detailed

ฯ In flat space, owing to the fact that partial derivatives commute, this transformation would actually leave one in the TT gauge. See [23] or Appendix C of [28] for a more detailed explanation. 
explanation, we present the basics and refer the interested reader to the literature for an in-depth account [7, 22].

The first step is to introduce new derivative operators $\tilde{\mathrm{P}}^{\prime}, \tilde{\partial}$ and $\tilde{\partial}^{\prime}=\overline{\tilde{\partial}}$ such that they commute with $\mathrm{P}$ when acting on quantities that $\mathrm{P}$ annihilates, ${ }^{+}$that is

$$
\left[\mathrm{P}, \tilde{\mathrm{P}}^{\prime}\right] x^{\circ}=0, \quad[\mathrm{P}, \tilde{\mathrm{\partial}}] x^{\circ}=0, \quad\left[\mathrm{P}, \tilde{\mathrm{\partial}}^{\prime}\right] x^{\circ}=0,
$$

where $[a, b]$ denotes the commutator between $a$ and $b$. The explicit form of the operators is given in Appendix B. The next step, the heart of Held's method, is to exploit the GHP equation $\mathrm{P} \rho=\rho^{2}$, and its complex conjugate $\mathrm{P} \bar{\rho}=\bar{\rho}^{2}$, to express everything as a polynomial in terms of $\rho$ and $\bar{\rho}$, with coefficients that are annihilated by $\mathrm{P}$. Held's method is then brought to completion by choosing four independent quantities to use as coordinates[8, 3]. In this work, we will not take this extra step. For type II spacetimes (and the accelerating C-metrics), this step has not been carried out, while for all remaining type D spacetimes, it has been carried through to completion[7, 22].

In a spacetime more general than type II, there is no possibility of having a repeated PND. When a repeated PND exists, we can appeal to the Goldberg-Sachs theorem [6] and set $\kappa=\sigma=\Psi_{0}=\Psi_{1}=0$ in (A.1-A.7). Following Held's partial integration of Petrov type II backgrounds[8], we also perform a null rotation (keeping $l^{a}$ fixed, but changing $n^{a}$ ) to set $\tau=0$. As a consequence, it follows from the GHP equations that $\tau^{\prime}=0$. Now we are in a position to address the question of when the full IRG can be imposed. First we apply the $l \cdot h$ gauge conditions (11) to (A.1-A.7). While most of the perturbed Einstein equations depend on several components of the metric perturbation, after imposing (11), the expression for $\mathcal{E}_{l l}$ depends only on $h_{m \bar{m}}$ and the $l l$-component of (8) simply becomes

$$
\{\mathrm{P}(\mathrm{P}-\rho-\bar{\rho})+2 \rho \bar{\rho}\} h_{m \bar{m}} \equiv\{(\mathrm{P}-2 \rho)(\mathrm{P}+\rho-\bar{\rho})\} h_{m \bar{m}}=8 \pi \mathcal{T}_{l l},
$$

in which the first form indicates that the equation is real, while the second form and its complex conjugate (which follow from the fact that $\mathrm{P} \rho=\rho^{2}$ and $\mathrm{P} \bar{\rho}=\bar{\rho}^{2}$ ) is the one we will use to integrate the equation below. If we had not made use of the Goldberg-Sachs theorem, there would be terms such as $\sigma \rho h_{\bar{m} \bar{m}}$ appearing in (18) and our argument would not hold. We immediately see that $\mathcal{T}_{l l}=0$ is necessary to satisfy the trace condition (12). Next we turn our attention to the question of whether the condition $\mathcal{E}_{l l}=0$, is sufficient to impose (12) using residual gauge freedom.

In order to address this question we will integrate $\mathcal{E}_{l l}=0$ and the residual gauge vector, given by the homogeneous form of (15). Full integration of the homogeneous form of (15) is carried out in Appendix B, but we will work through the integration of $\mathcal{E}_{l l}=0$ here to illustrate the method. We begin by rewriting (18), with the help of $\mathrm{P} \rho=\rho^{2}$ and its complex conjugate, as:

$$
\{(\mathrm{P}-2 \rho)(\mathrm{P}+\rho-\bar{\rho})\} h_{m \bar{m}}=\rho^{2} \mathrm{P}\left[\frac{\bar{\rho}}{\rho^{3}} \mathrm{P}\left(\frac{\rho}{\bar{\rho}} h_{m \bar{m}}\right)\right]=0 .
$$

\footnotetext{
+ Such quantities are denoted with the degree mark, ${ }^{\circ}$, as in $\mathrm{P} x^{\circ}=0$.
} 
Integrating once gives

$$
\mathrm{P}\left(\frac{\rho}{\bar{\rho}} h_{m \bar{m}}\right)=b^{\circ} \frac{\rho^{3}}{\bar{\rho}},
$$

and another integration leads to

$$
h_{m \bar{m}}=\bar{a}^{\circ} \frac{\bar{\rho}}{\rho}+\frac{1}{2} b^{\circ}(\rho+\bar{\rho}) .
$$

However, $h_{m \bar{m}}$ is, by definition, a real quantity, so we add the complex conjugate and use $b^{\circ}$ to represent a real quantity in the second term. The final result is that

$$
h_{m \bar{m}}=a^{\circ} \frac{\rho}{\bar{\rho}}+\bar{a}^{\circ} \frac{\bar{\rho}}{\rho}+b^{\circ}(\rho+\bar{\rho}) .
$$

Similarly, integration of (15), as carried out in Appendix B, leads to the following solution for the components of the residual gauge vector:

$$
\begin{aligned}
& \xi_{l}=\xi_{l}^{\circ}, \\
& \xi_{n}=\xi_{n}^{\circ}+\frac{1}{2}\left(\frac{1}{\rho}+\frac{1}{\bar{\rho}}\right) \tilde{\mathrm{P}}^{\prime} \xi_{l}^{\circ}+\frac{1}{2}\left(\Psi_{2}^{\circ} \rho+\bar{\Psi}_{2}^{\circ} \bar{\rho}\right) \xi_{l}^{\circ}, \\
& \xi_{m}=\frac{1}{\bar{\rho}} \xi_{m}^{\circ}-\tilde{\partial} \xi_{l}^{\circ}, \\
& \xi_{\bar{m}}=\frac{1}{\rho} \xi_{\bar{m}}^{\circ}-\tilde{\partial}^{\prime} \xi_{l}^{\circ},
\end{aligned}
$$

where $\Psi_{2}^{\circ}$ is related to the background curvature via $\Psi_{2}=\Psi_{2}^{\circ} \rho^{3}$. In order to use this residual gauge freedom to impose the full IRG, we return to the gauge transformation for $h_{m \bar{m}}$ (16) which becomes, after some manipulation (using (B.6-B.9) and (B.13)),

$h_{m \bar{m}}=\frac{\rho}{\bar{\rho}}\left[\tilde{\partial}^{\prime} \xi_{m}{ }^{\circ}+\tilde{\mathrm{P}}^{\prime} \xi_{l}^{\circ}\right]+\frac{\bar{\rho}}{\rho}\left[\tilde{\partial} \xi_{\bar{m}}^{\circ}+\tilde{\mathrm{P}}^{\prime} \xi_{l}^{\circ}\right]+(\rho+\bar{\rho})\left[-\frac{1}{2}\left(\tilde{\partial}^{\prime} \tilde{\partial}+\tilde{\partial} \tilde{\partial}^{\prime}-\rho^{\prime \circ}-\bar{\rho}^{\circ}\right) \xi_{l}{ }^{\circ}+\xi_{n}{ }^{\circ}\right]$.

In this form it is clear that we can impose the trace condition (12) of the full IRG if we choose our gauge vector so that

$$
\tilde{\partial}^{\prime} \xi_{m}{ }^{\circ}+\tilde{\mathrm{P}}^{\prime} \xi_{l}^{\circ}=a^{\circ}, \quad-\frac{1}{2}\left(\tilde{\check{\partial}}^{\prime} \tilde{\partial}+\tilde{\partial}^{\prime} \tilde{\partial}^{\prime}-\rho^{\prime \circ}-{\rho^{\prime}}^{\circ}\right) \xi_{l}^{\circ}+\xi_{n}{ }^{\circ}=b^{\circ} .
$$

We have now shown by construction that the condition $\mathcal{T}_{l l}=0$ is both necessary and sufficient for imposing the full IRG in a type II background. We turn next to discussing the complete extent of the residual gauge freedom in more detail.

\subsection{Remaining gauge freedom}

Although equations (25) involve three real degrees of freedom ( $a^{\circ}$ is complex), it turns out that only two real degrees of gauge freedom are required to fully remove any solution of (19) for the trace $h_{m \bar{m}}$. To see this we introduce the following identity:

$$
\frac{\rho}{\bar{\rho}}-\frac{\bar{\rho}}{\rho}=(\rho+\bar{\rho})\left(\frac{1}{\bar{\rho}}-\frac{1}{\rho}\right) \equiv(\rho+\bar{\rho}) \Omega^{\circ}
$$

which also defines $\Omega^{\circ}$, a quantity annihilated by $\mathrm{P}$. Then we can rewrite $(22)$ as

$$
h_{m \bar{m}}=\frac{1}{2}\left(a^{\circ}+\bar{a}^{\circ}\right)\left(\frac{\rho}{\bar{\rho}}+\frac{\bar{\rho}}{\rho}\right)+\left[\frac{1}{2}\left(a^{\circ}-\bar{a}^{\circ}\right) \Omega^{\circ}+b^{\circ}\right](\rho+\bar{\rho}) .
$$


In a similar fashion, we can rewrite $(24)$ as

$$
\begin{aligned}
h_{m \bar{m}}= & {\left[\frac{1}{2}\left(\tilde{\partial}^{\prime} \xi_{m}{ }^{\circ}+\tilde{\partial} \xi_{\bar{m}}{ }^{\circ}\right)+\tilde{\mathrm{P}}^{\prime} \xi_{l}^{\circ}\right]\left(\frac{\rho}{\bar{\rho}}+\frac{\bar{\rho}}{\rho}\right)+\left[\frac{1}{2}\left(\tilde{\partial}^{\prime} \xi_{m}{ }^{\circ}-\tilde{\partial} \xi_{\bar{m}}{ }^{\circ}\right) \Omega^{\circ}\right.} \\
& \left.-\frac{1}{2}\left(\tilde{\partial}^{\prime} \tilde{\partial}+\tilde{\partial} \tilde{\partial}^{\prime}-\rho^{\prime \circ}-\bar{\rho}^{\circ}\right) \xi_{l}^{\circ}+\xi_{n}{ }^{\circ}\right](\rho+\bar{\rho}),
\end{aligned}
$$

in which each coefficient in big square brackets is purely real. Now, suppose we have a particular solution for $\mathcal{E}_{l l}=0$, i.e., $a^{\circ}, \bar{a}^{\circ}$ and $b^{\circ}$ are fixed, and our task is to solve for the components of the gauge vector which removes this solution. By comparing (27) and (28) we see that, for any given $\xi_{m}{ }^{\circ}$ and $\xi_{\bar{m}}{ }^{\circ}$, we can fix $\xi_{l}{ }^{\circ}$ (up to a solution of $\tilde{\mathbf{P}}^{\prime} \xi_{l}{ }^{\circ}=0$ ) via

$$
\tilde{\mathrm{P}}^{\prime} \xi_{l}^{\circ}=\frac{1}{2}\left(a^{\circ}+\bar{a}^{\circ}\right)-\frac{1}{2}\left(\tilde{\partial}^{\prime} \xi_{m}^{\circ}+\tilde{\partial} \xi_{\bar{m}}^{\circ}\right),
$$

and we can fix $\xi_{n}{ }^{\circ}$ by setting

$\xi_{n}^{\circ}=\frac{1}{2}\left(a^{\circ}-\bar{a}^{\circ}\right) \Omega^{\circ}+b^{\circ}+\frac{1}{2}\left(\tilde{ð}^{\prime} \tilde{\partial}+\tilde{\partial} \tilde{\partial}^{\prime}-\rho^{\prime \circ}-\bar{\rho}^{\circ}\right) \xi_{l}^{\circ}-\frac{1}{2}\left(\tilde{\partial}^{\prime} \xi_{m}{ }^{\circ}-\tilde{\partial} \xi_{\bar{m}}{ }^{\circ}\right) \Omega^{\circ}$,

to completely eliminate the nonzero $h_{m \bar{m}}$, thus imposing the full IRG while still leaving two completely unconstrained degrees of gauge freedom, $\xi_{m}{ }^{\circ}$ and $\xi_{\bar{m}}{ }^{\circ}$. Once in the IRG, then (29) and (30), with $a^{\circ}, \bar{a}^{\circ}$ and $b^{\circ}$ set to zero and $\xi_{m}{ }^{\circ}$ and $\xi_{\bar{m}}{ }^{\circ}$ arbitrary, give the remaining components of a gauge vector preserving the IRG. It is currently unclear how to take advantage of this remaining gauge freedom to simplify the analysis of perturbations in the full IRG.

\section{Imposing the IRG in type D}

Type D background metrics are of considerable theoretical and observational interest since they include both the Schwarzschild and Kerr black hole spacetimes. Kinnersley first obtained all type D metrics by integrating the Newman-Penrose equations[11]. While the results of the previous section are general enough to encompass the special case of type D backgrounds, the tetrad choice we made (with $\tau=0$ ) is incompatible with the complete integration of the background field equations which is possible in type D spacetimes[7]. The complete integration requires that each of $l^{a}$ and $n^{a}$ be aligned with

one of the two PNDs. In that case we can exploit the full power of the Goldberg-Sachs theorem and its corollaries to set $\kappa=\kappa^{\prime}=\sigma=\sigma^{\prime}=\Psi_{0}=\Psi_{1}=\Psi_{3}=\Psi_{4}=0$, while maintaining $\tau \neq 0$ and $\tau^{\prime} \neq 0$. In this section we repeat the previous calculation with this different choice of tetrad.

The result of integrating $\mathcal{E}_{l l}=0$ is the same as in the case of a type II background, given in (22). The residual gauge vector, however, now has the following, more complex, 
form (details of the integration are given in Appendix B):

$$
\begin{aligned}
\xi_{l}= & \xi_{l}^{\circ}, \\
\xi_{n}= & \xi_{n}^{\circ}+\frac{1}{2} \Psi^{\circ} \xi_{l}^{\circ} \rho+\frac{1}{2} \bar{\Psi}^{\circ} \xi_{l}^{\circ} \bar{\rho}+\tau^{\circ} \bar{\tau}^{\circ} \xi_{l}^{\circ} \rho \bar{\rho}+\frac{1}{2} \pi^{\circ} \bar{\pi}^{\circ} \xi_{l}^{\circ}\left(\frac{1}{\rho^{2}}+\frac{1}{\bar{\rho}^{2}}\right) \\
& +\left[\frac{\pi^{\circ}}{\rho}\left(\tilde{\partial}+\bar{\alpha}^{\circ}\right)+\frac{\bar{\pi}^{\circ}}{\bar{\rho}}\left(\tilde{\partial}^{\prime}+\alpha^{\circ}\right)\right] \xi_{l}^{\circ}+\frac{1}{2}\left(\frac{1}{\rho}+\frac{1}{\bar{\rho}}\right) \tilde{\mathrm{p}}^{\prime} \xi_{l}^{\circ} \\
& -\left[\bar{\tau}^{\circ} \rho\left(\tilde{\partial}+\bar{\alpha}^{\circ}\right)+\tau^{\circ} \bar{\rho}^{\circ}\left(\tilde{\partial}^{\prime}+\alpha^{\circ}\right)\right] \xi_{l}^{\circ}+\bar{\tau}^{\circ} \xi_{m}{ }^{\circ} \frac{\rho}{\bar{\rho}}+\tau^{\circ} \xi_{\bar{m}}{ }^{\circ} \frac{\bar{\rho}}{\rho} \\
& -\pi^{\circ} \xi_{m}{ }^{\circ} \frac{1}{\bar{\rho}^{2}}-\bar{\pi}^{\circ} \xi_{\bar{m}}^{\circ} \frac{1}{\rho^{2}}-\alpha^{\circ} \xi_{m}{ }^{\circ} \frac{1}{\bar{\rho}}-\bar{\alpha}^{\circ} \xi_{\bar{m}}{ }^{\circ} \frac{1}{\rho}, \\
\xi_{m}= & \xi_{m}{ }^{\circ} \frac{1}{\bar{\rho}}-\bar{\pi}^{\circ} \xi_{l}^{\circ} \frac{1}{\rho}+\tau^{\circ} \xi_{l}^{\circ} \bar{\rho}-\left(\tilde{\partial}+\bar{\alpha}^{\circ}\right) \xi_{l}^{\circ}, \\
\xi_{\bar{m}}= & \xi_{\bar{m}}{ }^{\circ} \frac{1}{\rho}-\pi^{\circ} \xi_{l}^{\circ} \frac{1}{\bar{\rho}}+\bar{\tau}^{\circ} \xi_{l}^{\circ} \rho-\left(\tilde{\partial}^{\prime}+\alpha^{\circ}\right) \xi_{l}^{\circ},
\end{aligned}
$$

where the quantities $\Psi^{\circ}, \tau^{\circ}, \pi^{\circ}$ and $\alpha^{\circ}$ determine properties of the background spacetime. ${ }^{*}$ Now the gauge transformation for $h_{m \bar{m}}$ becomes

$$
\begin{aligned}
h_{m \bar{m}}= & \frac{\rho}{\bar{\rho}}\left[\tilde{\check{\partial}}^{\prime} \xi_{m}{ }^{\circ}+\tilde{\mathrm{P}}^{\prime} \xi_{l}^{\circ}-B^{\circ}\right]+\frac{\bar{\rho}}{\rho}\left[\tilde{\partial} \xi_{\bar{m}}{ }^{\circ}+\tilde{\mathrm{P}}^{\prime} \xi_{l}^{\circ}+B^{\circ}\right] \\
& +(\rho+\bar{\rho})\left[-\frac{1}{2}\left(\tilde{\check{\partial}}^{\prime} \tilde{\partial}+\tilde{\partial} \tilde{\partial}^{\prime}-\rho^{\prime \circ}-\bar{\rho}^{\circ}\right) \xi_{l}^{\circ}+\xi_{n}^{\circ}-A^{\circ}\right],
\end{aligned}
$$

where we have introduced (note that $B^{\circ}$ is purely imaginary)

$$
\begin{aligned}
& A^{\circ}=\frac{1}{2}\left\{2 \alpha^{\circ} \tilde{\partial}+\tilde{\partial}\left(\alpha^{\circ}\right)+\alpha^{\circ} \bar{\alpha}^{\circ}-\pi^{\circ} \tau^{\circ}\right\} \xi_{l}^{\circ}+\text { c.c. }, \\
& B^{\circ}=\frac{1}{4}\left\{4 \pi^{\circ} \tilde{\partial}+\tilde{\partial}\left(\pi^{\circ}\right)+5 \bar{\alpha}^{\circ} \pi^{\circ}-2 \pi^{\circ} \bar{\pi}^{\circ} \Omega^{\circ}\right\} \xi_{l}^{\circ}-\text { c.c. },
\end{aligned}
$$

with c.c. indicating the complex conjugate. Integration of the backgrounds where $\pi^{\circ} \neq 0$ and $\alpha^{\circ} \neq 0$ using the Held technique has not made its way into the literature and is beyond the scope of the present work. As a result, derivatives of $\pi^{\circ}$ and $\alpha^{\circ}$ appear in (33) but do no harm to our argument. Choosing any gauge vector that satisfies

$\tilde{\partial}^{\prime} \xi_{m}^{\circ}+\tilde{\mathrm{P}}^{\prime} \xi_{l}^{\circ}-B^{\circ}=a^{\circ}, \quad-\frac{1}{2}\left(\tilde{\partial}^{\prime} \tilde{\partial}+\tilde{\partial} \tilde{\partial}^{\prime}-\rho^{\prime \circ}-\bar{\rho}^{\circ}\right) \xi_{l}^{\circ}+\xi_{n}^{\circ}-A^{\circ}=b^{\circ}$,

will serve to impose the trace condition in the full IRG. Once again we have established that $\mathcal{T}_{l l}=0$ is both a necessary and sufficient condition for the existence of the full IRG. Note that by setting $\pi^{\circ}=\alpha^{\circ}=0$ (i.e. ignoring the C-metrics) in the background, $A^{\circ}=B^{\circ}=0$, and the result is virtually identical to (24) and (25). There is one simplification in that now $\bar{\rho}^{\circ}=\rho^{\circ}[22]$. The full extent of the remaining residual gauge freedom in (34) can be demonstrated along the same lines as used in section 4.1. As for the case of a type II background, it resides chiefly in the freely specifiable $\xi_{m}{ }^{\circ}$ and $\xi_{\bar{m}}{ }^{\circ}$.

* For example, $\pi^{\circ} \neq 0$ leads to the accelerating C-metrics. The condition $\pi^{\circ}=0$ implies $\alpha^{\circ}=0$ and so $\alpha^{\circ}$ is also related to parameters in the $\mathrm{C}$-metric. 


\section{Imposing the IRG in Schwarzschild spacetime}

We now demonstrate these results for Schwarzschild spacetime using conventional, spherically symmetric coordinates, in which the background metric takes the form:

$$
d s^{2}=f(r) d t^{2}-d r^{2} / f(r)-r^{2}\left(d \theta^{2}+\sin ^{2} \theta d \phi^{2}\right)
$$

where we have introduced $f(r)=(1-2 M / r)$. Metric perturbations, $h_{a b}(t, r, \theta, \phi)$, about the Schwarzschild geometry can be expressed in terms of the RW decomposition[20]. We decompose the angular dependence of these perturbations into spherical harmonics and the time dependence into constant frequency Fourier modes:

$$
h_{a b}(t, r, \theta, \phi)=\sum_{\ell, m, \omega} e^{-i \omega t} h_{a b}^{\ell m \omega}(r) \mathcal{O}_{a b}(\theta, \phi) Y_{\ell m}(\theta, \phi)
$$

where $\mathcal{O}_{a b}(\theta, \phi)$ is an angular operator (see [24] for details) and there is no sum here on the spacetime indices $\{a b\}$. With respect to rotation of the background coordinate system, $h_{t t}, h_{r r}$ and $h_{t r}$ transform as scalars, $\left\{h_{t \theta}, h_{t \phi}\right\}$ and $\left\{h_{r \theta}, h_{r \phi}\right\}$ transform as a pair of vectors on the 2 -sphere and $\left\{h_{\theta \theta}, h_{\theta \phi}, h_{\phi \phi}\right\}$ transforms as a symmetric covariant tensor on the 2-sphere.

It is well known that the components of the metric perturbation decouple into two classes, labeled as even and odd parity, according to their behavior under a parity transformation $P:(\theta \rightarrow \pi-\theta, \phi \rightarrow \pi+\phi)$. Out of the ten independent components of the metric perturbation for each mode (of specific $\{\ell, m, \omega\}$ ), the even parity perturbations, for which $P=(-1)^{\ell}$, have seven independent components and are given by $h_{a b}^{\text {even }}$,

$$
e^{-i \omega t}\left[\begin{array}{cccc}
f(r) H_{0}(r) & H_{1}(r) & { }^{e} h_{0}(r) \frac{\partial}{\partial \theta} & { }^{e} h_{0}(r) \frac{\partial}{\partial \phi} \\
\operatorname{sym} & H_{2}(r) / f(r) & { }^{e} h_{1}(r) \frac{\partial}{\partial \theta} & { }^{e} h_{1}(r) \frac{\partial}{\partial \phi} \\
\operatorname{sym} & \operatorname{sym} & r^{2}\left[K(r)+G(r) \frac{\partial^{2}}{\partial \theta^{2}}\right] & r^{2} G(r)\left[\frac{\partial^{2}}{\partial \theta \partial \phi}-\frac{\cos \theta}{\sin \theta} \frac{\partial}{\partial \phi}\right] \\
& & \text { sym } & r^{2} G(r)\left[\frac{\partial^{2}}{\partial \phi^{2}}+\frac{\sin 2 \theta}{2} \frac{\partial}{\partial \theta}\right] \\
\text { sym } & \text { sym } & & +r^{2} K(r) \sin ^{2} \theta
\end{array}\right] Y_{\ell m}(\Omega) .
$$

The odd parity perturbations, with $P=(-1)^{l+1}$, have three independent components, and are given by $h_{a b}^{\text {odd }}$,

$$
e^{-i \omega t}\left[\begin{array}{cccc}
0 & 0 & -{ }^{o} h_{0}(r) \frac{1}{\sin \theta} \frac{\partial}{\partial \phi} & { }^{o} h_{0}(r) \sin \theta \frac{\partial}{\partial \theta} \\
0 & 0 & -{ }^{o} h_{1}(r) \frac{1}{\sin \theta} \frac{\partial}{\partial \phi} & { }^{o} h_{1}(r) \sin \theta \frac{\partial}{\partial \theta} \\
\operatorname{sym} & \operatorname{sym} & h_{2}(r)\left[\frac{1}{\sin \theta} \frac{\partial^{2}}{\partial \theta \partial \phi}-\frac{\cos \theta}{\sin ^{2} \theta} \frac{\partial}{\partial \phi}\right] & \frac{1}{2} h_{2}(r)\left[\frac{1}{\sin \theta} \frac{\partial^{2}}{\partial \phi^{2}}+\right. \\
\text { sym } & \text { sym } & \operatorname{sym} & \left.\cos \theta \frac{\partial}{\partial \theta}-\sin \theta \frac{\partial^{2}}{\partial \theta^{2}}\right] \\
\left.\sin \theta \frac{\partial^{2}}{\partial \theta \partial \phi}-\cos \theta \frac{\partial}{\partial \phi}\right]
\end{array}\right] Y_{\ell m}(\Omega) .
$$

In the background spacetime, the Einstein tensor is identically zero. The perturbed Einstein tensor (which includes contributions from the metric perturbation $h_{a b}$ up to first order) transforms in the same way as the metric perturbations with respect to 
rotations on the 2-sphere. Hence, it has the same angular decomposition as the metric perturbation. For the even parity, we write the perturbed Einstein tensor, $\mathcal{E}_{a b}^{\text {even }}$, as

$$
e^{-i \omega t}\left[\begin{array}{cccc}
f(r) E_{1}(r) & E_{2}(r) & E_{4}(r) \frac{\partial}{\partial \theta} & E_{4}(r) \frac{\partial}{\partial \phi} \\
\operatorname{sym} & E_{3} / f(r) & E_{5}(r) \frac{\partial}{\partial \theta} & E_{5}(r) \frac{\partial}{\partial \phi} \\
\operatorname{sym} & \operatorname{sym} & r^{2}\left[E_{6}(r)+E_{7}(r) \frac{\partial^{2}}{\partial \theta^{2}}\right] & r^{2} E_{7}(r)\left[\frac{\partial^{2}}{\partial \theta \partial \phi}-\frac{\cos \theta}{\sin \theta} \frac{\partial}{\partial \phi}\right] \\
& & \operatorname{sym} & r^{2} E_{7}(r)\left[\frac{\partial^{2}}{\partial \phi^{2}}+\frac{\sin 2 \theta}{2} \frac{\partial}{\partial \theta}\right] \\
\operatorname{sym} & \operatorname{sym} & & +r^{2} E_{6}(r) \sin ^{2} \theta
\end{array}\right] Y_{\ell m}(\Omega),
$$

and for odd parity we write perturbed Einstein tensor, $\mathcal{E}_{a b}^{\text {odd }}$, as

$$
e^{-i \omega t}\left[\begin{array}{cccc}
0 & 0 & -F_{1}(r) \frac{1}{\sin \theta} \frac{\partial}{\partial \phi} & F_{1}(r) \sin \theta \frac{\partial}{\partial \theta} \\
0 & 0 & -F_{2}(r) \frac{1}{\sin \theta} \frac{\partial}{\partial \phi} & F_{2}(r) \sin \theta \frac{\partial}{\partial \theta} \\
\operatorname{sym} & \operatorname{sym} & F_{3}(r)\left[\frac{1}{\sin \theta} \frac{\partial^{2}}{\partial \theta \partial \phi}-\frac{\cos \theta}{\sin ^{2} \theta} \frac{\partial}{\partial \phi}\right] & \frac{1}{2} F_{3}(r)\left[\frac{1}{\sin \theta} \frac{\partial^{2}}{\partial \phi^{2}}+\right. \\
\text { sym } & \text { sym } & \operatorname{sym} & \left.\cos \theta \frac{\partial}{\partial \theta}-\sin \theta \frac{\partial^{2}}{\partial \theta^{2}}\right] \\
\text { sym } & & -F_{3}(r)\left[\sin \theta \frac{\partial^{2}}{\partial \theta \partial \phi}-\cos \theta \frac{\partial}{\partial \phi}\right]
\end{array}\right] Y_{\ell m}(\Omega) .
$$

The spherical symmetry and the static nature of the background geometry ensures that the perturbed Einstein equations decouple into individual modes of $\{\ell, m, \omega, P\}$. That is, each component of the perturbed $\mathcal{E}_{a b}$ belonging to a specific $\{\ell, m, \omega, P\}$ mode depends only on the metric perturbations of the same mode, $h_{a b}^{\ell m \omega P}$. Hence, it is generally sufficient to consider a single mode of the metric perturbation for our analysis.

We now impose the $l \cdot h$ gauge conditions $(9 a)$ on a specific mode of the perturbed metric. We have, $l^{a} h_{a b}=0$, where $l^{a}=(1 / f(r), 1,0,0)$ from equations (53) below, and $l^{a}$ is a repeated PND of the Schwarzschild background. For the odd parity perturbations, we can write $h_{0}$ in terms of $h_{1}$.

$$
{ }^{o} h_{0}(r)=-f(r){ }^{o} h_{1}(r) .
$$

For the even parity perturbations,

$$
H_{0}(r)=-H_{1}(r)=H_{2}(r), \quad \text { and } \quad{ }^{e} h_{0}(r)=-f(r){ }^{e} h_{1}(r) .
$$

The trace of the metric perturbations is a scalar with respect to rotation on a sphere of constant $r$ and constant $t$. Hence, it can be written as

$$
h_{a b} g^{a b}=\sum_{\ell, m, \omega, P} e^{-i \omega t} T^{\ell m \omega P}(r) Y_{\ell m}(\theta, \phi),
$$

Expanding the LHS gives,

$$
h_{a b} g^{a b}=\frac{1}{f(r)} h_{t t}-f(r) h_{r r}-\frac{1}{r^{2}} h_{\theta \theta}-\frac{1}{r^{2} \sin ^{2} \theta} h_{\phi \phi} .
$$

Note that the trace vanishes for the odd parity perturbations, while for the even parity the trace is equal to (we suppress labels $\{\ell, m, \omega, P\}$ when ambiguity is unlikely)

$$
T(r)=-[2 K(r)-\ell(\ell+1) G(r)]
$$


We have used the fact that the spherical harmonics are eigenfunctions of the angular momentum operator:

$$
\left[\frac{\partial^{2}}{\partial \theta^{2}}+\frac{1}{\sin ^{2} \theta} \frac{\partial^{2}}{\partial \phi^{2}}+\frac{\cos \theta}{\sin \theta} \frac{\partial}{\partial \theta}\right] Y_{\ell m}(\theta, \phi)=-\ell(\ell+1) Y_{\ell m}(\theta, \phi) .
$$

The perturbed Einstein tensor obtained in this gauge is given in Appendix C.

\subsection{Residual gauge freedom in the Schwarzschild geometry}

To determine the residual gauge freedom in the Schwarzschild background we require, as in (14),

$$
l^{a} h_{a b}=l^{a} \xi_{(a ; b)}
$$

Writing $l^{a} h_{a b}$ as $B_{b}$ and using the metric (35) to compute the covariant derivatives, gives

$$
\begin{aligned}
& B_{t}=\left[2 \xi_{t, t}-f^{\prime}(r) \xi_{t}\right] / f(r)+\xi_{r, t}+\xi_{t, r}-f^{\prime}(r) \xi_{r}, \\
& B_{r}=\left[\xi_{t, r}+\xi_{r, t}+f^{\prime}(r)\left\{\xi_{r}-\xi_{t} / f(r)\right\}\right] / f(r)+2 \xi_{r, r}, \\
& B_{\theta}=\left[\xi_{t, \theta}+\xi_{\theta, t}\right] / f(r)+\xi_{r, \theta}+\xi_{\theta, r}-2 \xi_{\theta} / r, \\
& B_{\phi}=\left[\xi_{t, \phi}+\xi_{\phi, t}\right] / f(r)+\xi_{r, \phi}+\xi_{\phi, r}-2 \xi_{\phi} / r .
\end{aligned}
$$

Gauge vectors, $\xi_{a}$, which correspond to residual gauge freedom, solve the above equations with $B_{a}=0$. Moreover, since we are going to deal with the metric perturbations of one single mode (specific $\{\ell, m, \omega, P\}$ ) at a time, we want $h_{a b}$ and $\xi_{a}$ to correspond to the same mode. This restricts the functional form of our gauge vector $\xi_{a}\left(x^{b}\right)$.

For even parity perturbations, we consider an even gauge vector of the form,

$$
\begin{aligned}
\xi_{t} & =-e^{-i \omega t} P(r) Y_{\ell m}(\theta, \phi), \\
\xi_{r} & =e^{-i \omega t} R(r) Y_{\ell m}(\theta, \phi), \\
\xi_{\theta} & =e^{-i \omega t} r S(r) \frac{\partial}{\partial \theta} Y_{\ell m}(\theta, \phi), \\
\xi_{\phi} & =e^{-i \omega t} r S(r) \frac{\partial}{\partial \phi} Y_{\ell m}(\theta, \phi) .
\end{aligned}
$$

For odd parity perturbations, we consider an odd gauge vector of the form,

$$
\begin{aligned}
& \xi_{t}=0, \\
& \xi_{r}=0, \\
& \xi_{\theta}=-e^{-i \omega t} Q(r) \frac{1}{\sin \theta} \frac{\partial}{\partial \phi} Y_{\ell m}(\theta, \phi), \\
& \xi_{\phi}=e^{-i \omega t} Q(r) \sin \theta \frac{\partial}{\partial \theta} Y_{\ell m}(\theta, \phi) .
\end{aligned}
$$


Inserting these forms of gauge vector into (44) and taking $B_{a}=0$, we arrive at equations for the residual gauge freedom. For the even parity gauge vector, we have

$0=e^{-i \omega t}\left[\left\{2 i \omega P(r)+f^{\prime}(r) P(r)\right\} / f(r)-i \omega R(r)-P^{\prime}(r)-f^{\prime}(r) R(r)\right] Y_{\ell m}(\theta, \phi)$,

$0=e^{-i \omega t}\left[\left\{f^{\prime}(r)[R(r)+P(r) / f(r)]-i \omega R(r)-P^{\prime}(r)\right\} / f(r)+2 R^{\prime}(r)\right] Y_{\ell m}(\theta, \phi)$,

$0=e^{-i \omega t}\left[-\{i \omega r S(r)+P(r)\} / f(r)+R(r)-S(r)+r S^{\prime}(r)\right] \frac{\partial}{\partial \theta} Y_{\ell m}(\theta, \phi)$,

$0=e^{-i \omega t}\left[-\{i \omega r S(r)+P(r)\} / f(r)+R(r)-S(r)+r S^{\prime}(r)\right] \frac{\partial}{\partial \phi} Y_{\ell m}(\theta, \phi)$.

For the odd parity gauge vector, we have

$$
\begin{aligned}
& 0=e^{-i \omega t}\left[i \omega Q(r) / f(r)-Q^{\prime}(r)+2 Q(r) / r\right] \frac{1}{\sin \theta} \frac{\partial}{\partial \phi} Y_{\ell m}(\theta, \phi), \\
& 0=-e^{-i \omega t}\left[i \omega Q(r) / f(r)-Q^{\prime}(r)+2 Q(r) / r\right] \sin \theta \frac{\partial}{\partial \theta} Y_{\ell m}(\theta, \phi) .
\end{aligned}
$$

These equations can be solved completely for the functions $P(r), R(r), S(r)$ and $Q(r)$ in terms of four arbitrary constants:

$$
\begin{aligned}
& P(r)=e^{i \omega r_{*}}\left[C_{1}-C_{2}(i \omega r+f(r))\right], \\
& R(r)=e^{i \omega r_{*}}\left[C_{1}-i \omega r C_{2}\right] / f(r), \\
& S(r)=e^{i \omega r_{*}}\left[C_{2}+C_{3} r\right], \text { and } \\
& Q(r)=e^{i \omega r_{*}} D r^{2},
\end{aligned}
$$

where we have introduced $r_{*}=r+2 M \ln (r / 2 M-1)$. These solutions correspond to the residual gauge freedom of the light-cone gauge in [18].

\subsection{Condition for the Trace to vanish}

The residual gauge freedom can be used to change the trace of the metric perturbation by a quantity $\operatorname{Tr}\left(\xi_{(a ; b)}\right)=g^{a b} \xi_{(a ; b)}$. For an odd parity perturbation, this quantity is easily seen to be zero. For an even parity perturbation, this quantity is evaluated to be

$\operatorname{Tr}\left(\xi_{(a ; b)}\right)=e^{-i \omega\left(t-r_{*}\right)}\left(2 i \omega C_{2}+\ell(\ell+1) C_{3}-\frac{2 C_{1}-\ell(\ell+1) C_{2}}{r}\right) Y_{\ell m}(\theta, \phi)$.

A particular linear combination, $E_{1}+2 E_{2}+E_{3}$, of the Einstein tensor components given in Appendix $\mathrm{C}$ is exactly $f(r) \mathcal{E}_{l l}$, and reduces to a second order operator acting only on the variable $T(r)$. For perturbations satisfying $\mathcal{T}_{l l}=0$, the $l l$-component of (8) gives:

$2 \mathcal{E}_{l l}=-T^{\prime \prime}(r)+\frac{2\left(i \omega r^{2}-r+2 M\right)}{r(r-2 M)} T^{\prime}(r)+\frac{2 i \omega(r-3 M)+\omega^{2} r^{2}}{(r-2 M)^{2}} T(r)=0$.

The solution to this equation is obtained in terms of two arbitrary constants $A, B$ :

$$
T(r)=e^{i \omega r_{*}}(A+B / r) .
$$

From (50), we already know the degrees of freedom that exist in the trace of the metric perturbation due to residual gauge freedom. One sees that the arbitrary constants $C_{1}, C_{2}, C_{3}$ can be chosen to exactly cancel $A$ and $B$. This analysis confirms 
that the residual gauge freedom can generally be exploited to set the trace of the metric perturbations to zero for perturbations with $\mathcal{T}_{l l}=0$, and verifies that $\mathcal{E}_{l l}=0$ is a sufficient condition for constructing an IRG. Once this has been done, there still exists one constant residual degree of gauge freedom per mode of metric perturbation (both even and odd), just as in sections 4 and 5. It is not clear how to fix these degrees of freedom in order to get some more useful analytical property of the metric perturbations.

Note that, in (50), when $\ell=0$, two of the four terms vanish identically. Next note that, for $\omega=0$, another term also vanishes. A time independent perturbation for which both $\ell$ and $\omega$ are zero, corresponds to a change in mass of the black hole. From (50), it would then appear that, for such a static mass perturbation, we can no longer cancel the A-term from the trace in (52). This is a mere artifact of our analysis and is not a fundamental obstacle. Use of the Fourier transform in $t$ is not permitted unless the perturbations belong in some suitable function space, say $L^{2}$. Polynomials would fail this test, but they are required in the gauge transformation for this case[18]. Allowing polynomials in $t$ and restoring the time derivative in (50) corrects this defect.

\subsection{Connection with the GHP Formulation}

Now that we have performed the same analysis for both a generic type D and the Schwarzschild backgrounds, we are in a position to make a direct comparison. For that purpose, we introduce a complete set of null tetrad vectors:

$l^{a}=(1 / f(r), 1,0,0), \quad n^{a}=\frac{1}{2}(1,-f(r), 0,0), \quad m^{a}=\frac{1}{\sqrt{2} r}(0,0,1, i / \sin \theta)$.

With this choice (introduced by Kinnersley[11]), the tetrad components of the residual gauge vector in the Schwarzschild spacetime become, after inserting (49) into (45) and (46):

$$
\begin{aligned}
& \xi_{l}=\xi_{a} a^{a}=e^{-i \omega\left(t-r_{*}\right)} C_{2} Y_{\ell m}(\theta, \phi), \\
& \xi_{n}=\xi_{a} n^{a}=-e^{-i \omega\left(t-r_{*}\right)}\left[C_{1}-C_{2}(i r \omega+f(r) / 2)\right] Y_{\ell m}(\theta, \phi), \\
& \xi_{m}=\xi_{a} m^{a}=\frac{1}{\sqrt{2}} e^{-i \omega\left(t-r_{*}\right)}\left[C_{2}+r C_{3}+i r D\right]\left[\frac{\partial}{\partial \theta}+\frac{i}{\sin \theta} \frac{\partial}{\partial \phi}\right] Y_{\ell m}(\theta, \phi) .
\end{aligned}
$$

Before carrying out the comparison, we need to express the null derivative operators in the Kinnersley tetrad (53). Acting on a scalar quantity, the operator P is given by

$$
\mathrm{P}=l^{a} \Theta_{a}=\frac{1}{f(r)} \frac{\partial}{\partial t}+\frac{\partial}{\partial r} .
$$

Therefore, in the Schwarzschild geometry, quantities annihilated by P have the form

$$
x^{\circ}=x^{\circ}\left(t-r_{*}, \theta, \phi\right) \text {. }
$$

Also, in the Schwarzschild geometry, $\bar{\rho}=\rho=-1 / r$ and $\tau=\tau^{\prime}=0$. So, when acting on scalars of spin-weight $s$, the operators $\tilde{\partial}$ and $\tilde{\partial}^{\prime}$ are (see (B.11) and (B.12) and also [5])

$$
\begin{aligned}
& \tilde{\partial}=\frac{\partial}{\bar{\rho}}=-r m^{a} \Theta_{a}=-\frac{(\sin \theta)^{s}}{\sqrt{2}}\left(\frac{\partial}{\partial \theta}+\frac{i}{\sin \theta} \frac{\partial}{\partial \phi}\right)(\sin \theta)^{-s}, \\
& \tilde{\partial}^{\prime}=\frac{\partial^{\prime}}{\rho}=-r \bar{m}^{a} \Theta_{a}=-\frac{(\sin \theta)^{-s}}{\sqrt{2}}\left(\frac{\partial}{\partial \theta}-\frac{i}{\sin \theta} \frac{\partial}{\partial \phi}\right)(\sin \theta)^{s} .
\end{aligned}
$$


Since $\bar{\Psi}_{2}=\Psi_{2}=\Psi^{\circ} \rho^{3}=-M / r^{3}$, we similarly have, for $\tilde{\mathrm{P}}^{\prime}$ acting on scalars of boostweight $b$ (see (B.10) in Appendix B, and [4]):

$$
\begin{aligned}
\tilde{\mathrm{P}}^{\prime} & =\mathrm{P}^{\prime}+\frac{(p+q) \Psi_{2}}{2 \rho}=n^{a} \Theta_{a}+b \Psi^{\circ} \rho^{2} \\
& =\frac{f(r)^{-b}}{2}\left(\frac{\partial}{\partial t}-f(r) \frac{\partial}{\partial r}+b \frac{2 M}{r^{2}}\right) f(r)^{b}=\frac{1}{2}\left(\frac{\partial}{\partial t}-f(r) \frac{\partial}{\partial r}\right) .
\end{aligned}
$$

Now, given that, in the Schwarzschild background, $\tau^{\circ}=\pi^{\circ}=\alpha^{\circ}=0$ and $\Psi^{\circ}=M$ we can write (31) as

$$
\begin{aligned}
& \xi_{l}=\xi_{l}^{\circ}, \\
& \xi_{n}=\xi_{n}^{\circ}-\frac{r}{2}\left(\frac{\partial}{\partial t}-f(r) \frac{\partial}{\partial r}+\frac{2 M}{r^{2}}\right) \xi_{l}^{\circ}, \\
& \xi_{m}=-r \xi_{m}^{\circ}+\frac{1}{\sqrt{2}}\left(\frac{\partial}{\partial \theta}+\frac{i}{\sin \theta} \frac{\partial}{\partial \phi}\right) \xi_{l}^{\circ} .
\end{aligned}
$$

Comparing (54) and (59), it is clear that $\sharp$

$$
\begin{aligned}
& \xi_{l}^{\circ}=e^{-i \omega\left(t-r_{*}\right)} C_{2} Y_{\ell m}(\theta, \phi), \\
& \xi_{n}^{\circ}=-e^{-i \omega\left(t-r_{*}\right)}\left(C_{1}-C_{2} / 2\right) Y_{\ell m}(\theta, \phi), \\
& \xi_{m}^{\circ}=-e^{-i \omega\left(t-r_{*}\right)} \frac{C_{3}+i D}{\sqrt{2}}\left(\frac{\partial}{\partial \theta}+\frac{i}{\sin \theta} \frac{\partial}{\partial \phi}\right) Y_{\ell m}(\theta, \phi) .
\end{aligned}
$$

To conclude, by comparing (52) and (22), and recalling $\bar{\rho}=\rho=-1 / r$, we see that with

$$
A=a^{\circ}+\bar{a}^{\circ}, \quad \text { and } B=-2 b^{\circ},
$$

the equivalence of the two formulations is established. Finally, with $\rho^{\prime \circ}=-\frac{1}{2}$, and using (B.2), (60) and (42) in (16), we can demonstrate its complete correspondence with (50).

\section{Discussion}

We have concentrated on Petrov type II spacetimes in this paper because they satisfy a minimum requirement necessary for the existence of a radiation gauge, namely the occurrence of a repeated PND. With our new form of the perturbed Einstein equations, use of NP methods has allowed us to treat this general class of spacetimes without either choosing coordinates or finding a metric. In this context, the Held technique has allowed us to exploit our form of the equations by enabling partial integration in solving $\mathcal{E}_{l l}=0$ while investigating the existence of the IRG. Additionally, the Held technique has allowed us to completely characterize the residual gauge freedom and use it in the radiation gauge construction. By explicit demonstration, this work establishes our new form of the perturbed Einstein equations as a powerful tool within perturbation theory, both in conjunction with the Held technique and otherwise.

For perturbations with $\mathcal{T}_{l l}=0$, our characterization of the residual gauge freedom is sufficiently complete that we can explicitly demonstrate the required gauge choice $\#$ To obtain these relations, we have used $r f^{\prime}(r)=1-f(r)$, which holds in the Schwarzschild spacetime. 
to remove any non-zero solution for the trace obtained via $\mathcal{E}_{l l}=0$. Thus, in type II spacetimes, radiation gauges can be established by a genuine gauge choice, even if only after a solution of $\mathcal{E}_{l l}=0$ is chosen.

There are subtle differences between the general type II case and the more restricted type D case, as there are also in the construction of Hertz potentials for the two cases. Stewart[23] writes out the type II case rather fully for an IRG. In this case, the perturbation in $\Psi_{0}$ is tetrad and gauge invariant, while the potential satisfies the adjoint (in the sense detailed by Wald[27]) of the $s=+2$ Teukolsky equation. Remarkably, in the type $\mathrm{D}$ case, this adjoint is actually the $s=-2$ Teukolsky equation, also satisfied by the gauge and tetrad invariant perturbation in $\Psi_{4}$. In the type II case, the adjoint equation is the same as in type $\mathrm{D}$, but $\Psi_{4}$ is no longer tetrad invariant. Compared to the type $\mathrm{D}$ result, the expression for $\Psi_{4}$ given by Stewart has many extra terms depending on $\kappa^{\prime}$ and $\sigma^{\prime}$, so presumably it does not satisfy the same equation as the potential. As a consequence, metric reconstruction would be restricted to being built around the perturbation for $\Psi_{0}$.

In the context of a specific type D background, Wald[25] has argued that mass and angular momentum perturbations are not given by any solution to the Teukolsky equations, and Stewart[23] has shown that these cannot be represented in a radiation gauge in terms of a potential. What we have done is identify the gauge freedom which remains in the fully satisfied radiation gauges, neither interfering with the radiation gauge prescription nor ruling out the possibility of mass and angular momentum perturbations. By realizing the explicit construction of the radiation gauges for type II background spacetimes and by identifying the remaining gauge freedom which they allow, we have, in a sense, completed a task initially embarked upon by Stewart[23], though in the different context of Hertz potentials.

\section{Acknowledgments}

This work has been supported in part by NSF Grants PHY-0245024 and PHY-0555484 with the University of Florida. L.R.P. acknowledges support from the Alumni Fellowship Program at the University of Florida during the early phases of the work reported here. K.S. acknowledges support from an IFT Summer Fellowship at the University of Florida during part of this work.

\section{Appendix A. The Perturbed Einstein Equations for a type II Background}

In this appendix we write the components of the perturbed Einstein tensor for an arbitrary type II background. We have assumed the PND is aligned with $l^{a}$ and made use of the Goldberg-Sachs theorem. Note that the equations for $\mathcal{E}_{l m}, \mathcal{E}_{n m}$ and $\mathcal{E}_{m m}$ are 
complex, so $\mathcal{E}_{l \bar{m}}=\overline{\mathcal{E}}_{l m}$ and so on:

$$
\begin{aligned}
& \mathcal{E}_{l l}=\left\{\left(\nearrow^{\prime}-\tau^{\prime}\right)\left(ð-\bar{\tau}^{\prime}\right)+\rho\left(\mathrm{P}^{\prime}+\rho^{\prime}-\bar{\rho}^{\prime}\right)-(\mathrm{P}-\rho) \rho^{\prime}+\Psi_{2}\right\} h_{l l} \\
& +\{-(\rho+\bar{\rho})(\mathrm{P}+\rho+\bar{\rho})+4 \rho \bar{\rho}\} h_{l n} \\
& +\left\{-(\mathrm{P}-3 \bar{\rho})\left(\mathrm{\partial}^{\prime}-\tau^{\prime}+\bar{\tau}\right)+\bar{\tau} \mathrm{P}-\bar{\rho} \mathrm{\partial}^{\prime}\right\} h_{l m} \\
& +\left\{-(\mathrm{P}-3 \rho)\left(ð+\tau-\bar{\tau}^{\prime}\right)+\tau \mathrm{P}-\rho ð\right\} h_{l \bar{m}} \\
& +\{\mathrm{P}(\mathrm{P}-\rho-\bar{\rho})+2 \rho \bar{\rho}\} h_{m \bar{m}}, \\
& \mathcal{E}_{n n}=\left\{2 \kappa^{\prime} \bar{\kappa}^{\prime}\right\} h_{l l} \\
& +\left\{\left(ð^{\prime}-\bar{\tau}\right)(ð-\tau)+\bar{\rho}^{\prime}(\mathrm{P}-\rho+\bar{\rho})-\left(\mathrm{P}^{\prime}-\bar{\rho}^{\prime}\right) \bar{\rho}+\bar{\Psi}_{2}+2 \bar{\rho} \bar{\rho}^{\prime}\right\} h_{n n} \\
& +\left\{-\left(\rho^{\prime}+\bar{\rho}^{\prime}\right)\left(\mathrm{P}^{\prime}+\rho^{\prime}+\bar{\rho}^{\prime}\right)+4 \rho^{\prime} \bar{\rho}^{\prime}-\left(\nearrow^{\prime}-2 \bar{\tau}\right) \bar{\kappa}^{\prime}-(ð-2 \tau) \kappa^{\prime}\right\} h_{l n} \\
& +\left\{\left(\mathrm{P}^{\prime}-\bar{\rho}^{\prime}\right) \kappa^{\prime}+\kappa^{\prime}\left(\mathrm{P}^{\prime}-\rho^{\prime}-\bar{\rho}^{\prime}\right)-\bar{\kappa}^{\prime} \sigma^{\prime}\right\} h_{l m} \\
& +\left\{\left(\mathrm{P}^{\prime}-\rho^{\prime}\right) \bar{\kappa}^{\prime}+\bar{\kappa}^{\prime}\left(\mathrm{P}^{\prime}-\bar{\rho}^{\prime}-\rho^{\prime}\right)-\kappa^{\prime} \bar{\sigma}^{\prime}\right\} h_{l \bar{m}} \\
& +\left\{-\left(\mathrm{P}^{\prime}-3 \rho^{\prime}\right)\left(\partial^{\prime}+\tau^{\prime}-\bar{\tau}\right)+\tau^{\prime} \mathrm{P}^{\prime}-\rho^{\prime} \partial^{\prime}-\kappa^{\prime} \mathrm{P}\right. \\
& \left.+(\mathrm{P}-2 \rho+\bar{\rho}) \kappa^{\prime}+\left(ð-3 \tau+\bar{\tau}^{\prime}\right) \sigma^{\prime}+ð\left(\sigma^{\prime}\right)-\Psi_{3}\right\} h_{n m} \\
& +\left\{-\left(\mathrm{P}^{\prime}-3 \bar{\rho}^{\prime}\right)\left(\partial+\bar{\tau}^{\prime}-\tau\right)+\bar{\tau}^{\prime} \mathrm{P}^{\prime}-\bar{\rho}^{\prime} \mathrm{\partial}-\bar{\kappa}^{\prime} \mathrm{P}\right. \\
& \left.+(\mathrm{P}-2 \bar{\rho}+\rho) \bar{\kappa}^{\prime}+\left(ð^{\prime}-3 \bar{\tau}+\tau^{\prime}\right) \bar{\sigma}^{\prime}+ð^{\prime}\left(\bar{\sigma}^{\prime}\right)-\bar{\Psi}_{3}\right\} h_{n \bar{m}} \\
& +\left\{-\left(\partial^{\prime}-2 \bar{\tau}\right) \kappa^{\prime}-\sigma^{\prime}\left(\mathrm{P}^{\prime}-\rho^{\prime}+\bar{\rho}^{\prime}\right)\right\} h_{m m} \\
& +\left\{-(\partial-2 \tau) \bar{\kappa}^{\prime}-\bar{\sigma}^{\prime}\left(\mathrm{P}^{\prime}-\bar{\rho}^{\prime}+\rho^{\prime}\right)\right\} h_{\bar{m} \bar{m}} \\
& +\left\{\mathrm{P}^{\prime}\left(\mathrm{P}^{\prime}-\rho^{\prime}-\bar{\rho}^{\prime}\right)+\kappa^{\prime}\left(\tau-\bar{\tau}^{\prime}\right)+\bar{\kappa}^{\prime}\left(\bar{\tau}-\tau^{\prime}\right)+2 \sigma^{\prime} \bar{\sigma}^{\prime}+2 \rho^{\prime} \bar{\rho}^{\prime}\right\} h_{m \bar{m}}, \\
& \mathcal{E}_{l n}=\frac{1}{2}\left\{\rho^{\prime}\left(\mathrm{P}^{\prime}-\rho^{\prime}\right)+\bar{\rho}^{\prime}\left(\mathrm{P}^{\prime}-\bar{\rho}^{\prime}\right)+\left(ð-2 \bar{\tau}^{\prime}\right) \kappa^{\prime}+\left(\partial^{\prime}-2 \tau^{\prime}\right) \bar{\kappa}^{\prime}+2 \sigma^{\prime} \bar{\sigma}^{\prime}\right\} h_{l l} \\
& +\frac{1}{2}\{\rho(\mathrm{P}-\rho)+\bar{\rho}(\mathrm{P}-\bar{\rho})\} h_{n n} \\
& +\frac{1}{2}\left\{-\left(\partial^{\prime}+\tau^{\prime}+\bar{\tau}\right)\left(\partial-\tau-\bar{\tau}^{\prime}\right)-\left(\partial^{\prime} \partial+3 \tau \tau^{\prime}+3 \bar{\tau} \bar{\tau}^{\prime}\right)+2\left(\bar{\tau}+\tau^{\prime}\right) \partial\right. \\
& \left.+(\mathrm{P}-2 \bar{\rho}) \rho^{\prime}+\left(\mathrm{P}^{\prime}-2 \rho^{\prime}\right) \bar{\rho}-\bar{\rho}^{\prime}(\mathrm{P}+\rho)-\rho\left(\mathrm{P}^{\prime}+\bar{\rho}^{\prime}\right)-\Psi_{2}-\bar{\Psi}_{2}\right\} h_{l n} \\
& +\frac{1}{2}\left\{\left(\mathrm{P}^{\prime}-2 \bar{\rho}^{\prime}\right)\left(\partial^{\prime}-\tau^{\prime}\right)+\bar{\tau}\left(\mathrm{P}^{\prime}+\rho^{\prime}+\bar{\rho}^{\prime}\right)-\tau^{\prime}\left(\mathrm{P}^{\prime}-\rho^{\prime}\right)\right. \\
& \left.-\left(2 \partial^{\prime}-\bar{\tau}\right) \bar{\rho}^{\prime}-(\mathrm{P}-2 \bar{\rho}) \kappa^{\prime}+\sigma^{\prime}\left(\tau-\bar{\tau}^{\prime}\right)\right\} h_{l m} \\
& +\frac{1}{2}\left\{\left(\mathrm{P}^{\prime}-2 \rho^{\prime}\right)\left(ð-\bar{\tau}^{\prime}\right)+\tau\left(\mathrm{P}^{\prime}+\bar{\rho}^{\prime}+\rho^{\prime}\right)-\bar{\tau}^{\prime}\left(\mathrm{P}^{\prime}-\bar{\rho}^{\prime}\right)\right. \\
& \left.-(2 ð-\tau) \rho^{\prime}-(\mathrm{P}-2 \rho) \bar{\kappa}^{\prime}+\bar{\sigma}^{\prime}\left(\bar{\tau}-\tau^{\prime}\right)\right\} h_{l \bar{m}} \\
& +\frac{1}{2}\left\{(\mathrm{P}-2 \rho)\left(\partial^{\prime}-\bar{\tau}\right)+\left(\tau^{\prime}+\bar{\tau}\right)(\mathrm{P}+\bar{\rho})-2\left(\partial^{\prime}-\tau^{\prime}\right) \rho-2 \bar{\tau} \mathrm{P}\right\} h_{n m} \\
& +\frac{1}{2}\left\{(\mathrm{P}-2 \bar{\rho})(\partial-\tau)+\left(\bar{\tau}^{\prime}+\tau\right)(\mathrm{P}+\rho)-2\left(ð-\bar{\tau}^{\prime}\right) \bar{\rho}-2 \tau \mathrm{P}\right\} h_{n \bar{m}} \\
& +\frac{1}{2}\left\{-\left(\partial^{\prime}-\bar{\tau}\right)\left(\partial^{\prime}-\tau^{\prime}\right)+\bar{\tau}\left(\bar{\tau}-\tau^{\prime}\right)-\sigma^{\prime} \rho\right\} h_{m m} \\
& +\frac{1}{2}\left\{-(\partial-\tau)\left(\partial-\bar{\tau}^{\prime}\right)+\tau\left(\tau-\bar{\tau}^{\prime}\right)-\bar{\sigma}^{\prime} \bar{\rho}\right\} h_{\bar{m} \bar{m}} \\
& +\frac{1}{2}\left\{\left(ð^{\prime}+\tau^{\prime}-\bar{\tau}\right)\left(\partial-\tau+\bar{\tau}^{\prime}\right)+\left(\partial^{\prime} \partial-\tau \tau^{\prime}-\bar{\tau} \bar{\tau}^{\prime}+\tau \bar{\tau}\right)-\left(\Psi_{2}+\bar{\Psi}_{2}\right)\right. \\
& +\left(\mathrm{P}^{\prime}-2 \rho^{\prime}\right) \bar{\rho}+(\mathrm{P}-2 \bar{\rho}) \rho^{\prime}+\rho\left(3 \mathrm{P}^{\prime}-2 \bar{\rho}^{\prime}\right)+\bar{\rho}^{\prime}(3 \mathrm{P}-2 \rho) \\
& \left.-2 \mathrm{P}^{\prime} \mathrm{P}+2 \rho \bar{\rho}^{\prime}+2{ }^{\prime}(\tau)-\tau \bar{\tau}\right\} h_{m \bar{m}},
\end{aligned}
$$


Radiation Gauges in type II spacetimes

$$
\begin{aligned}
& \mathcal{E}_{l m}=\frac{1}{2}\left\{\left(\mathrm{P}^{\prime}-\rho^{\prime}\right)\left(ð-\bar{\tau}^{\prime}\right)+\left(ð-\tau-2 \bar{\tau}^{\prime}\right) \bar{\rho}^{\prime}-(ð-\tau) \rho^{\prime}+\tau\left(\overline{\mathrm{P}}^{\prime}+\rho^{\prime}\right)\right. \\
& \left.+\bar{\sigma}^{\prime}\left(\partial^{\prime}-\tau^{\prime}+\bar{\tau}\right)+\bar{\Psi}_{3}+\bar{\rho} \bar{\kappa}^{\prime}\right\} h_{l l} \\
& +\frac{1}{2}\left\{-(\mathrm{P}-\rho+\bar{\rho})\left(\partial+\tau-\bar{\tau}^{\prime}\right)-\left(\partial-3 \tau+\bar{\tau}^{\prime}\right) \bar{\rho}-2 \rho \bar{\tau}^{\prime}\right\} h_{l n} \\
& +\frac{1}{2}\left\{-\left(\mathrm{P}^{\prime}+\bar{\rho}^{\prime}\right)(\mathrm{P}-2 \bar{\rho})+\rho\left(\mathrm{P}^{\prime}+2 \rho^{\prime}-2 \bar{\rho}^{\prime}\right)-4 \rho^{\prime} \bar{\rho}+2 \Psi_{2}\right. \\
& \left.+\left(ð^{\prime}+\bar{\tau}\right)\left(ð-2 \bar{\tau}^{\prime}\right)-\tau\left(ð^{\prime}+\tau^{\prime}-2 \bar{\tau}\right)-\tau^{\prime}\left(\tau-4 \bar{\tau}^{\prime}\right)\right\} h_{l m} \\
& +\frac{1}{2}\left\{-ð(\partial-2 \tau)-\bar{\sigma}^{\prime}(\mathrm{P}+2 \bar{\rho}-4 \rho)-2 \bar{\tau}^{\prime}\left(\tau-\bar{\tau}^{\prime}\right)\right\} h_{l \bar{m}} \\
& +\frac{1}{2}\{\mathrm{P}(\mathrm{P}-2 \rho)+2 \bar{\rho}(\rho-\bar{\rho})\} h_{n m} \\
& +\frac{1}{2}\left\{-(\mathrm{P}-\bar{\rho})\left(\chi^{\prime}-\tau^{\prime}+\bar{\tau}\right)+2 \bar{\tau} \bar{\rho}\right\} h_{m m} \\
& +\frac{1}{2}\left\{(\mathrm{P}+\rho-\bar{\rho})\left(\partial+\bar{\tau}^{\prime}-\tau\right)+2 \bar{\tau}^{\prime}(\mathrm{P}-2 \rho)-\left(ð-\tau-\bar{\tau}^{\prime}\right) \bar{\rho}+2 \rho \tau\right\} h_{m \bar{m}}, \\
& \mathcal{E}_{n \bar{m}}=\frac{1}{2}\left\{\left(\mathrm{P}^{\prime}-\rho^{\prime}\right) \kappa^{\prime}+\kappa^{\prime} \mathrm{P}^{\prime}+\bar{\kappa}^{\prime} \sigma^{\prime}\right\} h_{l l} \\
& +\frac{1}{2}\left\{(\mathrm{P}-\rho+\bar{\rho})\left(\partial^{\prime}-\bar{\tau}\right)-\left(\partial^{\prime}-2 \tau^{\prime}+\bar{\tau}\right) \rho+\tau^{\prime}(\mathrm{P}-\bar{\rho})\right\} h_{n n} \\
& +\frac{1}{2}\left\{\left(-\left(\mathrm{P}^{\prime}-\rho^{\prime}+\bar{\rho}^{\prime}\right)\left(ð^{\prime}+\tau^{\prime}-\bar{\tau}\right)-\left(ð^{\prime}-3 \tau^{\prime}+\bar{\tau}\right) \bar{\rho}^{\prime}+\left(ð-\tau+\bar{\tau}^{\prime}\right) \sigma^{\prime}\right.\right. \\
& \left.-2 \sigma^{\prime} ð-\Psi_{3}-2 \rho^{\prime} \bar{\tau}\right\} h_{l n} \\
& +\left\{\sigma^{\prime}\left(\rho^{\prime}-2 \bar{\rho}^{\prime}\right)-\kappa^{\prime}\left(\tau^{\prime}-2 \bar{\tau}\right)+\frac{1}{2} \Psi_{4}\right\} h_{l m} \\
& +\frac{1}{2}\left\{\left(\mathrm{P}^{\prime}\left(\mathrm{P}^{\prime}-2 \rho^{\prime}\right)+-\kappa^{\prime}\left(ð-2 \tau+2 \bar{\tau}^{\prime}\right)+\bar{\kappa}^{\prime}\left({ }^{\prime}-4 \tau^{\prime}+2 \bar{\tau}\right)\right.\right. \\
& \left.+2 \bar{\rho}^{\prime}\left(\rho^{\prime}-\bar{\rho}^{\prime}\right)+2 \sigma^{\prime} \bar{\sigma}^{\prime}\right\} h_{l \bar{m}} \\
& +\frac{1}{2}\left\{-ð^{\prime}\left(\partial^{\prime}-2 \tau^{\prime}\right)+\sigma^{\prime}(\mathrm{P}-2 \rho+2 \bar{\rho})-2 \bar{\tau}\left(\tau^{\prime}-\bar{\tau}\right)\right\} h_{n m} \\
& +\frac{1}{2}\left\{-\left(\mathrm{P}^{\prime}-\bar{\rho}^{\prime}\right)(\mathrm{P}+2 \bar{\rho})+\rho\left(\mathrm{P}^{\prime}-2 \bar{\rho}^{\prime}\right)+2 \bar{\rho}^{\prime}(\mathrm{P}-\rho)-\Psi_{2}-2 \bar{\Psi}_{2}\right. \\
& \left.+\left(\partial^{\prime}-3 \bar{\tau}\right) ð+\bar{\tau}^{\prime}\left(2 ð^{\prime}-\bar{\tau}+4 \tau^{\prime}\right)-\tau\left(\partial^{\prime}-2 \bar{\tau}\right)\right\} h_{n \bar{m}} \\
& +\frac{1}{2}\left\{-\left(\partial^{\prime}-\tau^{\prime}\right) \sigma^{\prime}-\sigma^{\prime} \partial^{\prime}\right\} h_{m m} \\
& +\frac{1}{2}\left\{-\left(\mathrm{P}^{\prime}-\bar{\rho}^{\prime}\right)\left(ð-\tau+\bar{\tau}^{\prime}\right)+2 \bar{\tau}^{\prime} \bar{\rho}^{\prime}-\bar{\kappa}^{\prime}(\mathrm{P}-2 \rho+2 \bar{\rho})+ð^{\prime}\left(\bar{\sigma}^{\prime}\right)-\bar{\tau} \bar{\sigma}^{\prime}\right\} h_{\bar{m} \bar{m}} \\
& +\frac{1}{2}\left\{\left(\mathrm{P}^{\prime}+\rho^{\prime}-\bar{\rho}^{\prime}\right)\left(\chi^{\prime}-\tau^{\prime}+\bar{\tau}\right)+2 \bar{\tau}\left(\mathrm{P}^{\prime}-2 \rho^{\prime}\right)-\left(\chi^{\prime}-\tau^{\prime}-\bar{\tau}\right) \bar{\rho}^{\prime}+2 \rho^{\prime} \tau^{\prime}\right. \\
& \left.+\left(ð-\tau-\bar{\tau}^{\prime}\right) \sigma^{\prime}+\sigma^{\prime} ð-\kappa^{\prime} \mathrm{P}-\Psi_{3}\right\} h_{m \bar{m}}, \\
& \mathcal{E}_{m m}=\left\{\left(\mathrm{P}^{\prime}-2 \rho^{\prime}\right) \bar{\sigma}^{\prime}+\bar{\kappa}^{\prime}\left(\partial+\tau-\bar{\tau}^{\prime}\right)\right\} h_{l l} \\
& +\left\{-ð\left(ð-\tau-\bar{\tau}^{\prime}\right)-2 \tau \bar{\tau}^{\prime}+\bar{\sigma}^{\prime}(\rho-\bar{\rho})\right\} h_{l n} \\
& +\left\{\left(\mathrm{P}^{\prime}-\rho^{\prime}\right)\left(\partial-\bar{\tau}^{\prime}\right)-\left(ð-\tau-\bar{\tau}^{\prime}\right) \rho^{\prime}+\tau\left(\mathrm{P}^{\prime}+\rho^{\prime}-\bar{\rho}^{\prime}\right)-(\mathrm{P}-2 \bar{\rho}) \bar{\kappa}^{\prime}\right. \\
& \left.-\bar{\tau}^{\prime}\left(\mathrm{P}+\bar{\rho}^{\prime}\right)+\bar{\tau} \bar{\sigma}^{\prime}-\bar{\Psi}_{3}\right\} h_{l m} \\
& +\left\{-\left(ð-\tau-\bar{\tau}^{\prime}\right) \bar{\sigma}^{\prime}-\bar{\sigma}^{\prime}(\partial-\tau)\right\} h_{l \bar{m}} \\
& +\left\{(\mathrm{P}-\bar{\rho})(ð-\tau)-\left(ð-\tau-\bar{\tau}^{\prime}\right) \bar{\rho}-\tau(\mathrm{P}+\rho)+\bar{\tau}^{\prime}(\mathrm{P}-\rho+\bar{\rho})\right\} h_{n m} \\
& +\left\{-\left(\mathrm{P}^{\prime}-\rho^{\prime}\right)(\mathrm{P}-\bar{\rho})+(ð-\tau) \tau^{\prime}-\tau\left(\partial^{\prime}+\tau^{\prime}-\bar{\tau}\right)+\Psi_{2}\right\} h_{m m} \\
& +\left\{(\mathrm{P}-2 \bar{\rho}) \bar{\sigma}^{\prime}+\left(\tau+\bar{\tau}^{\prime}\right) \partial+\left(\tau-\bar{\tau}^{\prime}\right)^{2}\right\} h_{m \bar{m}},
\end{aligned}
$$




$$
\begin{aligned}
& \mathcal{E}_{m \bar{m}}=\frac{1}{2}\left\{\mathrm{P}^{\prime}\left(\mathrm{P}^{\prime}-\rho^{\prime}-\bar{\rho}^{\prime}\right)+2 \rho^{\prime} \bar{\rho}^{\prime}+\kappa^{\prime}\left(\tau-\bar{\tau}^{\prime}\right)-\bar{\kappa}^{\prime}\left(\bar{\tau}-\tau^{\prime}\right)+2 \sigma^{\prime} \bar{\sigma}^{\prime}\right\} h_{l l} \\
& +\frac{1}{2}\{\mathrm{P}(\mathrm{P}-\rho-\bar{\rho})+2 \rho \bar{\rho}\} h_{n n} \\
& +\frac{1}{2}\left\{-\left(\mathrm{P}^{\prime}+\rho^{\prime}-\bar{\rho}^{\prime}\right)(\mathrm{P}-\rho+\bar{\rho})-\mathrm{P}^{\prime}(\mathrm{P}+\rho)+\rho\left(\mathrm{P}^{\prime}+\rho^{\prime}-\bar{\rho}^{\prime}\right)-\bar{\Psi}_{2}\right. \\
& +\left(\partial^{\prime}-\bar{\tau}\right)\left(ð-\tau-\bar{\tau}^{\prime}\right)+ð^{\prime} \partial-\left(ð-2 \bar{\tau}^{\prime}\right) \tau^{\prime}-\bar{\tau}\left(2 ð+\bar{\tau}^{\prime}\right) \\
& \left.-2 \tau\left(\partial^{\prime}-\bar{\tau}\right)+2 \tau^{\prime} \bar{\tau}^{\prime}+\bar{\rho} \bar{\rho}^{\prime}\right\} h_{l n} \\
& +\frac{1}{2}\left\{-\left(\mathrm{P}^{\prime}-2 \rho^{\prime}\right)\left(ð^{\prime}-2 \bar{\tau}\right)+\bar{\tau}\left(\mathrm{P}^{\prime}+2 \rho^{\prime}-2 \bar{\rho}^{\prime}\right)+2\left(ð-\bar{\tau}^{\prime}\right) \sigma^{\prime}-\sigma^{\prime} \precsim\right. \\
& \left.-2 \tau^{\prime} \bar{\rho}^{\prime}-2 \kappa^{\prime}(\rho-\bar{\rho})-\Psi_{3}\right\} h_{l m} \\
& +\frac{1}{2}\left\{-\left(\mathrm{P}^{\prime}-2 \bar{\rho}^{\prime}\right)(ð-2 \tau)+\tau\left(\mathrm{P}^{\prime}+2 \bar{\rho}^{\prime}-2 \bar{\rho}\right)+2\left(ð^{\prime}-\tau^{\prime}\right) \bar{\sigma}^{\prime}-\bar{\sigma}^{\prime} \partial^{\prime}\right. \\
& \left.-2 \bar{\tau}^{\prime} \rho^{\prime}-2 \bar{\kappa}^{\prime}(\bar{\rho}-\rho)-\bar{\Psi}_{3}\right\} h_{l \bar{m}} \\
& +\frac{1}{2}\left\{-(\mathrm{P}-2 \bar{\rho})\left(ð^{\prime}-2 \tau^{\prime}\right)+\tau^{\prime}(\mathrm{P}-2 \rho-2 \bar{\rho})-2 \rho \bar{\tau}+4 \tau^{\prime} \bar{\rho}\right\} h_{n m} \\
& +\frac{1}{2}\left\{-(\mathrm{P}-2 \rho)\left(ð-2 \bar{\tau}^{\prime}\right)+\bar{\tau}^{\prime}(\mathrm{P}-2 \bar{\rho}-2 \rho)-2 \bar{\rho} \tau+4 \bar{\tau}^{\prime} \rho\right\} h_{n \bar{m}} \\
& +\frac{1}{2}\left\{-\bar{\tau}\left(ð^{\prime}-\bar{\tau}\right)-\tau^{\prime}\left(ð^{\prime}-\tau^{\prime}\right)-(\mathrm{P}-2 \bar{\rho}) \sigma^{\prime}\right\} h_{m m} \\
& +\frac{1}{2}\left\{-\tau(\partial-\tau)-\bar{\tau}^{\prime}\left(\partial-\bar{\tau}^{\prime}\right)-(\mathrm{P}-2 \rho) \bar{\sigma}^{\prime}\right\} h_{\bar{m} \bar{m}} \\
& +\frac{1}{2}\left\{2 \mathrm{P}^{\prime} \mathrm{P}-\left(\mathrm{P}^{\prime}-\bar{\rho}^{\prime}\right) \bar{\rho}-(\mathrm{P}-\rho) \rho^{\prime}-\rho\left(\mathrm{P}^{\prime}-\rho^{\prime}+\bar{\rho}^{\prime}\right)-\bar{\rho}^{\prime}(\mathrm{P}+\rho-\bar{\rho})\right. \\
& -\left(\nearrow^{\prime}-2 \tau^{\prime}\right) \bar{\tau}^{\prime}+\tau\left(ð^{\prime}+2 \bar{\tau}\right)-\tau^{\prime}\left(ð-\bar{\tau}^{\prime}\right)+\bar{\tau}(ð+\tau)-ð^{\prime}(\tau) \\
& \left.-\Psi_{2}-\bar{\Psi}_{2}\right\} h_{m \bar{m}} \text {. }
\end{aligned}
$$

\section{Appendix B. Integration à la Held $[7,8,22]$}

We provide details of the integration that lead to (23) and (31). As it turns out, the type II calculation is actually much simpler than the the type D calculation because it uses a tetrad in which $\tau=\tau^{\prime}=0$. Therefore we will work out the type $\mathrm{D}$ calculation in detail and the type II result mostly follows by setting certain quantities to zero, as indicated below.

We will need some results (and their complex conjugates) from the integration of the type D background:

$$
\begin{aligned}
\tilde{\partial}^{\prime} \rho= & -\pi^{\circ} \frac{\rho}{\bar{\rho}}-\alpha^{\circ} \rho-\bar{\tau}^{\circ} \rho^{2}, \\
\rho^{\prime}= & \rho^{\prime \circ} \bar{\rho}-\frac{1}{2} \Psi^{\circ} \rho^{2}-\left(\tilde{\partial} \bar{\tau}^{\circ}+\frac{1}{2} \Psi^{\circ}\right) \rho \bar{\rho}-\tau^{\circ} \bar{\tau}^{\circ} \rho^{2} \bar{\rho}+\bar{\tau}^{\circ} \bar{\pi}^{\circ} \rho \\
& +\bar{\tau}^{\circ} \bar{\alpha}^{\circ} \rho^{2}+\frac{1}{2} \pi^{\circ} \bar{\pi}^{\circ} \bar{\rho}\left(\frac{1}{\rho^{2}}+\frac{1}{\bar{\rho}^{2}}\right)+\frac{1}{2} \bar{\rho}\left(\frac{1}{\rho}+\frac{1}{\bar{\rho}}\right)\left(\tilde{\partial}+\bar{\alpha}^{\circ}\right) \pi^{\circ}, \\
\tau= & -\bar{\pi}^{\circ}-\bar{\alpha}^{\circ} \rho+\tau^{\circ} \rho \bar{\rho}, \\
\tau^{\prime}= & -\pi^{\circ}-\bar{\tau}^{\circ} \rho^{2}, \\
\Psi_{2}= & \Psi^{\circ} \rho^{3} .
\end{aligned}
$$

As noted in the text, $\pi^{\circ} \neq 0$ leads to the accelerating C-metrics, which we include for full generality. Henceforth the corresponding quantities in type II spacetimes can be obtained by setting $\tau^{\circ}=\pi^{\circ}=\alpha^{\circ} \Rightarrow 0$ and $\Psi^{\circ} \Rightarrow \Psi_{2}{ }^{\circ} \dagger \dagger$ in the type D result. Thus, in $\dagger \dagger$ This arises from the fact that in type D spacetimes there is only one non-vanishing Weyl scalar, $\Psi_{2}$. 
type II spacetimes we have

$$
\begin{aligned}
\rho^{\prime} & =\rho^{\prime \circ} \bar{\rho}-\frac{1}{2} \Psi_{2}^{\circ}\left(\rho^{2}+\rho \bar{\rho}\right), \\
\tau & =0, \\
\tau^{\prime} & =0, \\
\Psi_{2} & =\Psi_{2}^{\circ} \rho^{3},
\end{aligned}
$$

the equation for $\tilde{\partial}^{\prime} \rho$ not following from the limiting process mentioned above. Note that the quantity $\tilde{\partial}^{\prime} \rho$ is never used in any of the integrations we perform in the type II background spacetime. We will also need the definitions of the new operators:

$$
\begin{aligned}
& \tilde{\mathrm{P}}^{\prime}=\mathrm{P}^{\prime}-\bar{\tau} \tilde{\partial}-\tau \tilde{\partial}^{\prime}+\tau \bar{\tau}\left(\frac{p}{\bar{\rho}}+\frac{q}{\rho}\right)+\frac{1}{2}\left(\frac{p \Psi_{2}}{\rho}+\frac{q \bar{\Psi}_{2}}{\bar{\rho}}\right), \\
& \tilde{\partial}=\frac{\partial}{\bar{\rho}}+\frac{q \tau}{\rho}, \\
& \tilde{\partial}^{\prime}=\frac{ð^{\prime}}{\rho}+\frac{p \bar{\tau}}{\bar{\rho}},
\end{aligned}
$$

where $p$ and $q$ label the GHP type of the quantity being acted on. Additionally, in sections 4 and 5 we make use of the commutator

$$
\begin{aligned}
{\left[\tilde{\partial}, \tilde{\partial}^{\prime}\right]=} & \frac{\bar{\rho}^{\prime}-\rho^{\prime}}{\rho \bar{\rho}} \mathbf{P}+\left(\frac{1}{\bar{\rho}}-\frac{1}{\rho}\right) \tilde{\mathbf{P}}^{\prime}+p\left\{\frac{\rho^{\prime}}{\bar{\rho}}+\frac{1}{2} \Psi_{2}\left(\frac{1}{\rho}+\frac{1}{\bar{\rho}}\right)+\tilde{\partial}\left(\frac{\bar{\tau}}{\bar{\rho}}\right)\right\} \\
& -q\left\{\frac{\bar{\rho}^{\prime}}{\rho}+\frac{1}{2} \bar{\Psi}_{2}\left(\frac{1}{\rho}+\frac{1}{\bar{\rho}}\right)+\tilde{\partial}^{\prime}\left(\frac{\tau}{\rho}\right)\right\},
\end{aligned}
$$

which is valid in type $\mathrm{D}$ and (with $\tau=0$ ) type II spacetimes.

We now begin with

$$
\mathrm{P} \xi_{l}=0,
$$

which integrates trivially to give

$$
\xi_{l}=\xi_{l}^{\circ}
$$

With this information in hand, we can now integrate the equation governing $\xi_{m}$ :

$$
(\mathrm{P}+\bar{\rho}) \xi_{m}+\left(ð+\bar{\tau}^{\prime}\right) \xi_{l}=0 .
$$

Rewriting the $\mathrm{P}$ piece and using (B.11) with $p=1$ leads to

$$
\frac{1}{\bar{\rho}} \mathrm{P}\left(\bar{\rho} \xi_{m}\right)+\bar{\tau}^{\prime} \xi_{l}+\bar{\rho} \tilde{\mathrm{d}} \xi_{l}-\frac{\bar{\rho} \tau}{\rho} \xi_{l}=0,
$$

which, after substituting (B.3), the complex conjugate of (B.4) and (B.15) along with some rearranging, yields

$$
\mathrm{P}\left(\bar{\rho} \xi_{m}\right)=-\bar{\pi}^{\circ} \xi_{l}^{\circ}\left(\frac{\bar{\rho}^{2}}{\rho}-\bar{\rho}\right)+2 \tau^{\circ} \xi_{l}^{\circ} \bar{\rho}^{3}-\bar{\rho}^{2}\left(\tilde{\partial}+\bar{\alpha}^{\circ}\right) \xi_{l}^{\circ}
$$

In type II spacetimes, however, both $\Psi_{3}$ and $\Psi_{4}$ are in general also nonzero. Though we do not refer to any of the other Weyl scalars in this work, we would like maintain agreement with the standard conventions. 
Integration then gives us

$$
\xi_{m}=\xi_{m}{ }^{\circ} \frac{1}{\bar{\rho}}-\bar{\pi}^{\circ} \xi_{l}^{\circ} \frac{1}{\rho}+\tau^{\circ} \xi_{l}^{\circ} \bar{\rho}-\left(\tilde{\widetilde{\partial}}+\bar{\alpha}^{\circ}\right) \xi_{l}^{\circ},
$$

and the solution for $\xi_{\bar{m}}$ then follows from complex conjugation

$$
\xi_{\bar{m}}=\xi_{\bar{m}}^{\circ} \frac{1}{\rho}-\pi^{\circ} \xi_{l}^{\circ} \frac{1}{\bar{\rho}}+\bar{\tau}^{\circ} \xi_{l}^{\circ} \rho-\left(\tilde{\partial}^{\prime}+\alpha^{\circ}\right) \xi_{l}^{\circ} .
$$

Finally, we are in a position to deal with $\xi_{n}$, by writing

$$
\mathrm{P}^{\prime} \xi_{l}+\mathrm{P} \xi_{n}+\left(\tau+\bar{\tau}^{\prime}\right) \xi_{\bar{m}}+\left(\bar{\tau}+\tau^{\prime}\right) \xi_{m}=0,
$$

in terms of Held's operators ((B.1), (B.3) and (B.4)) as

$$
\begin{aligned}
\mathrm{P} \xi_{n}+\tilde{\mathrm{P}}^{\prime} \xi_{l}+\bar{\tau} \widetilde{\mathrm{d}} \xi_{l}+\tau \widetilde{\partial}^{\prime} \xi_{l}-\tau \bar{\tau}\left(\frac{1}{\rho}+\frac{1}{\bar{\rho}}\right) \xi_{l} \\
\quad-\frac{1}{2}\left(\frac{\Psi_{2}}{\rho}+\frac{\bar{\Psi}_{2}}{\bar{\rho}}\right) \xi_{l}+\left(\tau+\bar{\tau}^{\prime}\right) \xi_{\bar{m}}+\left(\bar{\tau}+\tau^{\prime}\right) \xi_{m}=0 .
\end{aligned}
$$

Substituting (B.3), (B.4), (B.5), (B.15), (B.19) and (B.20), rearranging terms and letting the dust settle leads to

$$
\begin{aligned}
& \mathrm{P} \xi_{n}=-\tilde{\mathrm{P}}^{\prime} \xi_{l}^{\circ}+\frac{1}{2} \Psi^{\circ} \xi_{l}^{\circ} \rho^{2}+\frac{1}{2} \bar{\Psi}^{\circ} \xi_{l}^{\circ} \bar{\rho}^{2}-\pi^{\circ} \bar{\pi}^{\circ} \xi_{l}^{\circ}\left(\frac{1}{\rho}+\frac{1}{\bar{\rho}}\right) \\
& +\tau^{\circ} \bar{\tau}^{\circ} \xi_{l}^{\circ}\left(\rho^{2} \bar{\rho}+\rho \bar{\rho}^{2}\right)-\left[\bar{\tau}^{\circ} \rho^{2}\left(\tilde{\partial}+\bar{\alpha}^{\circ}\right)+\tau^{\circ} \bar{\rho}^{2}\left(\tilde{\partial}^{\prime}+\alpha^{\circ}\right)\right] \xi_{l}^{\circ} \\
& -\left[\pi^{\circ}\left(\tilde{\widetilde{\partial}}+\bar{\alpha}^{\circ}\right)+\bar{\pi}^{\circ}\left(\tilde{\check{\partial}}^{\prime}+\alpha^{\circ}\right)\right] \xi_{l}^{\circ}+2 \pi^{\circ} \xi_{m}{ }^{\circ} \frac{1}{\bar{\rho}}+2 \bar{\pi}^{\circ} \xi_{\bar{m}}^{\circ} \frac{1}{\rho} \\
& +\tau^{\circ} \xi_{\bar{m}}^{\circ}\left(\frac{\bar{\rho}^{2}}{\rho}-\bar{\rho}\right)+\bar{\tau}^{\circ} \xi_{m}^{\circ}\left(\frac{\rho^{2}}{\bar{\rho}}-\rho\right)+\alpha^{\circ} \xi_{m}^{\circ}+\bar{\alpha}^{\circ} \xi_{\bar{m}}^{\circ} \text {. }
\end{aligned}
$$

Integration then results in

$$
\begin{aligned}
\xi_{n}= & \xi_{n}{ }^{\circ}+\frac{1}{2} \Psi^{\circ} \xi_{l}^{\circ} \rho+\frac{1}{2} \bar{\Psi}^{\circ} \xi_{l}^{\circ} \bar{\rho}+\tau^{\circ} \bar{\tau}^{\circ} \xi_{l}^{\circ} \rho \bar{\rho}+\frac{1}{2} \pi^{\circ} \bar{\pi}^{\circ} \xi_{l}^{\circ}\left(\frac{1}{\rho^{2}}+\frac{1}{\bar{\rho}^{2}}\right) \\
& +\left[\frac{\pi^{\circ}}{\rho}\left(\tilde{\partial}+\bar{\alpha}^{\circ}\right)+\frac{\bar{\pi}^{\circ}}{\bar{\rho}}\left(\tilde{\partial}^{\prime}+\alpha^{\circ}\right)\right] \xi_{l}^{\circ}+\frac{1}{2}\left(\frac{1}{\rho}+\frac{1}{\bar{\rho}}\right) \tilde{\mathrm{P}}^{\prime} \xi_{l}^{\circ} \\
& -\left[\bar{\tau}^{\circ} \rho\left(\tilde{\partial}+\bar{\alpha}^{\circ}\right)+\tau^{\circ} \bar{\rho}\left(\tilde{\partial}^{\prime}+\alpha^{\circ}\right)\right] \xi_{l}^{\circ}+\bar{\tau}^{\circ} \xi_{m}{ }^{\circ} \frac{\rho}{\bar{\rho}}+\tau^{\circ} \xi_{\bar{m}}{ }^{\circ} \frac{\bar{\rho}}{\rho} \\
& -\pi^{\circ} \xi_{m}{ }^{\circ} \frac{1}{\bar{\rho}^{2}}-\bar{\pi}^{\circ} \xi_{\bar{m}}{ }^{\circ} \frac{1}{\rho^{2}}-\alpha^{\circ} \xi_{m}{ }^{\circ} \frac{1}{\bar{\rho}}-\bar{\alpha}^{\circ} \xi_{\bar{m}}{ }^{\circ} \frac{1}{\rho},
\end{aligned}
$$

and our task is complete.

\section{Appendix C. Perturbed Einstein Tensor in Schwarzschild spacetime}

We list the components of the Einstein tensor expressed in terms of the metric perturbations in the gauge $l^{a} h_{a b}=0$. The independent components of the even parity metric perturbations in this gauge are, using (38): $H(r) \equiv H_{0}(r), h(r) \equiv{ }^{e} h_{0}(r), K(r)$ 
and $G(r)$. The trace is given by $T(r)=-2 K(r)+\ell(\ell+1) G(r)$. The components of the Einstein tensor $E_{1} \ldots E_{7}$ are calculated to be:

$$
\begin{aligned}
& E_{1}(r)=-\frac{(r-2 M)}{2 r} T^{\prime \prime}(r)-\frac{(3 r-5 M)}{2 r^{2}} T^{\prime}(r)-\frac{[\ell(\ell+1)-2]}{2 r^{2}} K(r) \\
& -\frac{\ell(\ell+1)}{r^{2}} h^{\prime}(r)-\frac{\ell(\ell+1)(r-3 M)}{(r-2 M) r^{3}} h(r) \\
& -\frac{(r-2 M)}{r^{2}} H^{\prime}(r)-\frac{[\ell(\ell+1)+2]}{2 r^{2}} H(r) \\
& E_{2}(r)=\frac{i \omega}{2} T^{\prime}(r)+\frac{i \omega(r-3 M)}{2 r(r-2 M)} T(r)+\frac{[\ell(\ell+1)+2 i r \omega]}{2 r^{2}} H(r) \\
& +\frac{\ell(\ell+1)}{2 r^{2}} h^{\prime}(r)+\frac{\ell(\ell+1)\left(i \omega r^{2}-2 M\right)}{2(r-2 M) r^{3}} h(r) \\
& E_{3}(r)=\frac{(r-M)}{2 r^{2}} T^{\prime}(r)+\frac{r \omega^{2}}{2(r-2 M)} T(r)+\frac{\ell(\ell+1)\left(r-M-i \omega r^{2}\right)}{r^{3}(r-2 M)} h(r) \\
& +\frac{(r-2 M)}{r^{2}} H^{\prime}(r)-\frac{2 i r \omega}{r^{2}} H(r)-\frac{[\ell(\ell+1)-2]}{2 r^{2}}[H(r)-K(r)], \\
& E_{4}(r)=\frac{(r-2 M)}{2 r} h^{\prime \prime}(r)-\frac{i \omega}{2} h^{\prime}(r)+\frac{\left[2 M(r-2 M)-i \omega r^{2}(r-3 M)\right]}{r^{3}(r-2 M)} h(r) \\
& +\frac{(r-2 M)}{2 r} H^{\prime}(r)+\frac{\left(2 M-i \omega r^{2}\right)}{2 r^{2}} H(r)-\frac{i \omega}{2}[K(r)-G(r)], \\
& E_{5}(r)=-\frac{i \omega r}{2(r-2 M)} h^{\prime}(r)-\frac{\left[2(r-2 M)(1-i \omega r)+r^{3} \omega^{2}\right]}{2 r(r-2 M)^{2}} h(r) \\
& -\frac{1}{2} H^{\prime}(r)-\frac{\left(2 M-i \omega r^{2}\right)}{2 r(r-2 M)} H(r)+\frac{1}{2}\left[K^{\prime}(r)-G^{\prime}(r)\right], \\
& E_{6}(r)=\frac{(r-2 M)}{2 r} H^{\prime \prime}(r)+\frac{(1-i \omega r)}{r} H^{\prime}(r)-\frac{i \omega\left(2 r-2 M-i \omega r^{2}\right)}{2 r(r-2 M)} H(r) \\
& +\ell(\ell+1)\left[\frac{(r-2 M)}{2 r} G^{\prime \prime}(r)+\frac{(r-M)}{r^{2}} G^{\prime}(r)+\frac{r \omega^{2}}{2(r-2 M)} G(r)\right] \\
& -\left[\frac{(r-2 M)}{2 r} K^{\prime \prime}(r)+\frac{(r-M)}{r^{2}} K^{\prime}(r)+\frac{r \omega^{2}}{2(r-2 M)} K(r)\right] \\
& +\frac{\ell(\ell+1)}{r^{2}} h^{\prime}(r)-\frac{i \omega \ell(\ell+1)}{r(r-2 M)} h(r), \\
& E_{7}=\frac{(r-2 M)}{2 r} G^{\prime \prime}(r)+\frac{(r-M)}{r^{2}} G^{\prime}(r)+\frac{r \omega^{2}}{2(r-2 M)} G(r) \\
& +\frac{1}{r^{2}} h^{\prime}(r)-\frac{i \omega}{r(r-2 M)} h(r) .
\end{aligned}
$$


The independent components of the odd parity perturbations in this gauge are, using (37): $h(r) \equiv{ }^{o} h_{0}(r)$ and $H(r) \equiv h_{2}(r)$. The components of Einstein tensor $F_{1} \ldots F_{3}$ are calculated to be

$$
\begin{aligned}
F_{1}(r)= & \frac{r-2 M}{2 r} h^{\prime \prime}(r)-\frac{i \omega}{2} h^{\prime}(r)+\frac{i \omega[\ell(\ell+1)-2]}{4 r^{2}} H(r) \\
& -\frac{i \omega r^{3}+[\ell(\ell+1) / 2-i \omega 3 M] r^{2}-[\ell(\ell+1)+2] r M+4 M^{2}}{r^{3}(r-2 M)} h(r), \\
F_{2}(r)= & -\frac{[\ell(\ell+1)-2]}{4 r^{2}} H^{\prime}(r)-\frac{i \omega r}{2(r-2 M)} h^{\prime}(r)+\frac{[\ell(\ell+1)-2]}{2 r^{3}} H(r) \\
& -\frac{r^{3} \omega^{2} / 2-(r-2 M)[i \omega r-1+\ell(\ell+1) / 2]}{r(r-2 M)^{2}} h(r), \\
F_{3}(r)= & \frac{(r-2 M)}{2 r} H^{\prime \prime}(r)-\frac{(r-3 M)}{r^{2}} H^{\prime}(r)-h^{\prime}(r) \\
& +\frac{i \omega r}{(r-2 M)} h(r)+\frac{\left(r^{4} \omega^{2} / 2+r^{2}-6 M r+8 M^{2}\right)}{r^{3}(r-2 M)} H(r) .
\end{aligned}
$$

\section{References}

[1] P. L. Chrzanowski, Vector potential and metric perturbations of a rotating black hole, Phys. Rev. D11 (1975), 2042-2062.

[2] J. M. Cohen and L. S. Kegeles, Space-time perturbations, Phys. Lett. 54A (1975), 5-7.

[3] Brian Edgar, Integration methods within existing tetrad formalisms in general relativity, Gen. Rel. Grav. 24 (1992), 1267-1295.

[4] R. Geroch, A. Held, and R. Penrose, A space-time calculus based on pairs of null directions, J. Math. Phys. 14 (1973), 874-881.

[5] J. N. Goldberg, A. J. MacFarlane, E. T. Newman, F. Rohrlich, and E. C. G. Sudarshan, Spin-s spherical harmonics and edth, J. Math. Phys. 8 (1967), 2155-2161.

[6] J. N. Goldberg and R. K. Sachs, A theorem on Petrov types, Acta Phys. Polonica, (Suppl. 13) 22 (1962), 13-23.

[7] Alan Held, A formalism for the investigation of algebraically special metrics. I, Commun. Math. Phys. 37 (1974), 311-326.

[8] _ A formalism for the investigation of algebraically special metrics. II, Commun. Math. Phys. 44 (1975), 211-222.

[9] Jacek Jezierski, Energy and angular momentum of the weak gravitational waves on the Schwarzschild background: Quasilocal gauge-invariant formulation, Gen. Rel. Grav. 31 (1999), $1855-1890$.

[10] L. S. Kegeles and J. M. Cohen, Constructive procedure for perturbations of space-times, Phys. Rev. D19 (1979), 1641-1664.

[11] William Kinnersley, Type D vacuum metrics, J. Math. Phys. 10 (1969), 1195-1203.

[12] Carlos O. Lousto and Bernard F. Whiting, Reconstruction of black hole metric perturbations from Weyl curvature, Phys. Rev. D66 (2002), 024026.

[13] Vincent Moncrief, Gravitational perturbations of spherically symmetric systems. I: The exterior problem, Annals of Physics 88 (1974), 323-342.

[14] Ezra Newman and Roger Penrose, An approach to gravitational radiation by a method of spin coefficients, J. Math. Phys. 3 (1962), 566-578. 
[15] Brien C. Nolan, Physical interpretation of gauge invariant perturbations of spherically symmetric space-times, Phys. Rev. D70 (2004), 044004.

[16] Amos Ori, Reconstruction of inhomogeneous metric perturbations and electromagnetic fourpotentials in Kerr spacetime, Phys. Rev. D67 (2003), 124010.

[17] R. Penrose and W. Rindler, Spinors and spacetime. Volume 1, Cambridge University Press, New York, 1984.

[18] Brent Preston and Eric Poisson, A light-cone gauge for black-hole perturbation theory, Phys. Rev. D74 (2006), 064010.

[19] Richard H. Price, Nonspherical perturbations of relativistic gravitational collapse. II. Integer-spin, zero-rest-mass fields, Phys. Rev. D5 (1972), 2439-2454.

[20] Tullio Regge and John A. Wheeler, Stability of a Schwarzschild singularity, Phys. Rev. 108 (1957), 1063-1069.

[21] J. Scherk and J. H. Schwarz, Gravitation in the light-cone gauge, Gen. Rel. Grav. 6 (1975), 537-550.

[22] J. M. Stewart and M. Walker, Perturbations of space-times in general relativity, Proc. Roy. Soc. Lond. A341 (1974), 49-74.

[23] John M. Stewart, Hertz-Bromwich-Debye-Whittaker-Penrose potentials in general relativity, Proc. Roy. Soc. Lond. A367 (1979), 527-538.

[24] Kip S. Thorne, Multipole expansions of gravitational radiation, Rev. Mod. Phys. 52 (1980), 299-339

[25] Robert M. Wald, On perturbations of a Kerr black hole, J. Math. Phys. 14 (1973), 1453-1461.

[26] R. M. Wald, General relativity, The University of Chicago Press, Chicago, 1984.

[27] Robert M. Wald, Construction of solutions of gravitational, electromagnetic, or other perturbation equations from solutions of decoupled equations, Phys. Rev. Lett. 41 (1978), 203-206.

[28] B. F. Whiting and L. R. Price, Metric reconstruction from Weyl scalars, Class. Quantum Grav. 22 (2005), S589-S604. 